\title{
Existence, uniqueness and removable singularities for nonlinear partial differential equations in geometry
}

\author{
F. Reese Harvey and H. Blaine Lawson, Jr.
}

\begin{abstract}
This paper surveys some recent results on existence, uniqueness and removable singularities for fully nonlinear differential equations on manifolds. The discussion also treats restriction theorems and the strong Bellman principle.
\end{abstract}

\section{Contents}

1. Introduction 103

2. Subequations - A Geometric Approach 111

3. Jet Equivalence of Subequations 118

4. Monotonicity. 123

5. Comparison and Strict Approximation 128

6. Removable Singularities 131

7. Boundary Convexity 133

8. The Dirichlet Problem 136

9. Restriction Theorems 143

10. Convex Subequations and the Strong Bellman Principle 145

11. Applications to Almost Complex Manifolds 147

Appendix A. A Pocket Dictionary 148

Appendix B. Examples of Basic Monotonicity Cones 150

References $\quad 152$

\section{Introduction}

Calibrated geometries are considered generalizations of Kähler geometry. They resemble Kähler geometry in having large families of distinguished

Partially supported by the N.S.F. 
subvarieties determined by a fixed differential form. On the other hand, they seemed at first to be unlike Kähler geometry in having no suitable analogue of holomorphic functions. However, it was realized several years ago that the analogues of plurisubharmonic functions do exist (in abundance) on any calibrated manifold, and a potential theory was developed in this context $\left[\mathrm{HL}_{2,3}\right]$. This led us naturally to the study of "maximal" or "extremal" functions, the analogues of solutions to the homogeneous complex MongeAmpère equation, first considered by Bremermann [B] and Walsh [W] and later developed, in the inhomogeneous case, by Bedford-Taylor [BT* $\mathrm{BT}_{*}$ and others. The techniques and results developed in our study turned out to have substantial applications outside of calibrated geometry - in particular to many of the highly degenerate elliptic equations which appear naturally in geometry.

This paper is a survey of those techniques and results. We will address questions of existence and uniqueness for the Dirichlet Problem, the question of removable singularities for solutions and subsolutions, and the problem of restriction. The techniques apply broadly to fully nonlinear (second-order) equations in geometry, and in particular, to those which arise "universally" on riemannian, hermitian, or calibrated manifolds. A number of examples and applications will be discussed, including a proof of the Pali Conjecture on almost complex manifolds. Many more examples appear in the references.

It is conventional in discussing nonlinear differential equations to introduce the notions of a subsolution and supersolution, and define a solution to be a function which is both. In this paper we adopt an intrinsic approach by specifying a subset $F$ of constraints on the value of a function and its derivatives. The classical subsolutions are defined to be the $C^{2}$-functions $u$ whose 2-jet $\left(u, D u, D^{2} u\right)$ lies in $F$ at each point. The set $F$ will be called a subequation, and the functions $u$ with $\left(u, D u, D^{2} u\right) \in F$ are called $F$ subharmonic.

The notion of supersolution is captured by the dual subequation

$$
\widetilde{F} \equiv-\{\sim \operatorname{Int} F\}=\sim\{-\operatorname{Int} F\}
$$

and classical solutions $u$ are just those where $u$ is $F$-subharmonic and $-u$ is $\widetilde{F}$-subharmonic. They have the property that $\left(u, D u, D^{2} u\right) \in \partial F$ at each point, since $\partial F=F \cap(\sim \widetilde{F})$, and they will be called $F$-harmonic functions.

The simplest example is the Laplace equation, where $F=\left\{\operatorname{tr}\left(D^{2} u\right) \geq\right.$ $0\}=\widetilde{F}$.

The most basic example is the Monge-Ampère subequation $\mathcal{P}=$ $\left\{D^{2} u \geq 0\right\}$ with $\partial \mathcal{P} \subset\left\{\operatorname{det} D^{2} u=0\right\}$. The dual $\widetilde{\mathcal{P}}$-subharmonics are the subaffine functions (see 2.1.8).

Adopting this point of view brings out an internal duality:

$$
\widetilde{\widetilde{F}}=F
$$


and enables the roles of $F$ and $\widetilde{F}$ to be interchanged in the analysis. This symmetry is often enlightening. It is particularly so when discussing the boundary geometry necessary for solving the Dirichlet problem.

A dictionary relating this approach to the more classical one is given in Appendix A.

The first step in our analysis is to extend the notion of $F$-subharmonicity to general upper semi-continuous $[-\infty, \infty)$-valued functions. This is done in $\S 2$ where it is noted that these generalized $F$-subharmonic functions enjoy essentially all the useful properties of classical subharmonic functions. However, for this to be meaningful, $F$ must satisfy a certain positivity condition, corresponding to weak ellipticity. We also require a negativity condition, corresponding to weak "properness".

For the sake of clarity our exposition will often jump between the two extreme cases:

(1) Constant coefficient (parallel) subequations in $\mathbf{R}^{n}$, and

(2) General subequations on manifolds.

In fact, for many equations of interest in geometry and, in particular, those which are the principal focus of this survey, these two cases are directly related by the notion of jet-equivalence, introduced in $\S 3$. This basic concept plays a fundamental role in our work. Jet-equivalence is a certain transformation of all the variables. It can often be quite radical - turning mild equations into nasty ones, homogeneous equations into inhomogeneous ones, etc.

As stated, many important nonlinear equations on manifolds are locally jet-equivalent, in local coordinates, to constant coefficient equations. In this case the results of Slodkowski $\left[\mathrm{S}_{1}\right]$ and Jensen $\left[\mathrm{J}_{1}\right]$, and methods of viscosity theory [CIL], [C] can be applied to prove local weak comparison, and therefore global weak comparison - the first main step in the analysis of the Dirichlet Problem.

This leads to another concept of basic importance here: that of a monotonicity cone, introduced in $\S 4$. It gives the approximation tools needed to promote weak comparison to full comparison (see Definition 5.1) which, together with appropriate boundary geometry, yields both uniqueness and existence for the Dirichlet Problem. A subequation $M$ is called a monotonicity cone for a subequation $F$ if

$$
F+M \subset F
$$

and each fibre $M_{x}$, for $x \in X$, is a convex cone with vertex at the origin. One has that

$$
F+M \subset F \quad \Longleftrightarrow \quad \widetilde{F}+M \subset \widetilde{F},
$$

so a monotonicity cone for $F$ is also one for $\widetilde{F}$.

Monotonicity cones play a role in the theory of removable singularities. For $M$ as above, we define a closed subset $E \subset X$ to be $M$-polar if 
$E=\{x: \psi(x)=-\infty\}$ for some $M$-subharmonic function which is smooth on $X-E$.

If $M$ is a monotonicity cone for a subequation $F$, then $M$-polar sets are removable for $F$-subharmonic and $F$-harmonic functions on $X$.

(See Theorems 6.2.1 and 6.2.2.) This applies, for example, to all branches of the complex Monge-Ampère equation (see 2.1.10). Moreover, if a constant pure second-order subequation $F$ in $\mathbf{R}^{n}$ is $M$-monotone, where $M \equiv \mathcal{P}(p) \subset$ $\operatorname{Sym}^{2}\left(\mathbf{R}^{n}\right)$ is defined in terms of the ordered eigenvalues by $\lambda_{1}(A)+\cdots+$ $\lambda_{[p]}(A)+(p-[p]) \lambda_{p+1}(A) \geq 0$, then

$$
\begin{gathered}
\text { any closed subset of locally finite Hausdorff } p-2 \text { measure } \\
\text { is removable for } F \text { and } \widetilde{F} \text {. }
\end{gathered}
$$

This applies to the calibration case. It generalizes certain results in [CLN], $[\mathrm{AGV}]$ and $\left[\mathrm{La}_{*}\right]$.

Monotonicity cones also play a key role in comparison. The monotonicity condition (1.1.1) is equivalent to

$$
F+\widetilde{F} \subset \widetilde{M}
$$

For many basic monotonicity cones, the $\widetilde{M}$-subharmonic functions satisfy the Zero Maximum Principle (see Appendix B). In such cases, comparison (see 5.1) comes down to an addition theorem: if $u$ is $F$-subharmonic and $v$ is $\widetilde{F}$-subharmonic, then $u+v$ is $\widetilde{M}$ subharmonic.

There is a last ingredient needed for the Dirichlet Problem - the necessary boundary geometry. Associated to each subequation $F$, there is a notion of strict $F$-convexity for oriented hypersurfaces. There are certain equations, like the k-Laplacian for $1<k \leq \infty$ (see 7.4(a)), for which all hypersurfaces are strictly $F$-convex. This convexity is defined in terms of the asymptotic geometry of $F$ at infinity (see $\S 7$ ). It is quite often easy to compute, and it can be expressed directly in terms of the second fundamental form.

This notion of boundary convexity implies existence, via the Perron process, once comparison has been established.

If comparison holds for a subequation $F$ on a manifold $X$, then the Dirichlet Problem is uniquely solvable for F-harmonic functions on every domain $\Omega \subset X$ with smooth boundary which is strictly $F$ and $\widetilde{F}$ convex.

Unique solvability for the Dirichlet Problem means that for every $\varphi \in$ $C(\partial \Omega)$, there exists a unique $u \in C(\bar{\Omega})$ such that

$$
\left.u\right|_{\Omega} \in F(\Omega) \quad \text { and }\left.\quad u\right|_{\partial \Omega}=\varphi
$$

This theorem combines with results discussed above to prove the following general result.

THEOREM 8.1.2. Let $F$ be a subequation with monotonicity cone $M$. Suppose that: 
(i) F is locally affinely jet-equivalent to a constant coefficient subequation, and

(ii) $X$ carries a smooth strictly $M$-subharmonic function.

Then existence and uniqueness hold for the Dirichlet problem for Fharmonic functions on any domain $\Omega \subset \subset X$ whose boundary is both strictly $F$ - and $\widetilde{F}$-convex.

The global condition (ii) is essential for a result of this generality. For example, suppose $X$ is a riemannian manifold and $F \equiv\{$ Hess $u \geq 0\}$, where Hess $u$ is the riemannian hessian. Given a domain $\Omega \subset \subset X$ with strictly convex boundary, one can completely change the geometry and topology in the interior of $\Omega$ without affecting the boundary. The subequation $F$ continues to satisfy (i), but solutions to the Dirichlet Problem won't exist unless (ii) is satisfied. Another good example is the complex analogue $F=$ $\mathcal{P}^{\mathbf{C}}$ on an almost complex hermitian manifold (the homogeneous complex Monge-Ampère equation). Here condition (ii) amounts to the hypothesis that $X$ carries at least one strictly plurisubharmonic function.

In homogeneous spaces one can apply a trick of Walsh [W] to establish existence without uniqueness.

Theorem 8.1.3. Let $X=G / H$ be a riemannian homogeneous space and suppose that $F \subset J^{2}(X)$ is a subequation which is invariant under the natural action of $G$ on $J^{2}(X)$. Let $\Omega \subset \subset X$ be a connected domain whose boundary is both $F$ and $\widetilde{F}$ strictly convex. Then existence holds for the Dirichlet problem for $F$-harmonic functions on $\Omega$.

These results apply to a wide spectrum of equations. Many examples have been discussed in $\left[\mathrm{HL}_{4,6,7}\right]$ and are summarized in $\S 2$ below.

- (Constant Coefficients). Theorem 8.1.3 establishes existence for any constant coefficient subequation $F$ in $\mathbf{R}^{n}$, and uniqueness also follows, by 8.1 .2 , whenever $F$ has monotonicity cone $M$ and there exists a strictly $M$-subharmonic function on $\bar{\Omega}$. If $F$ is pure secondorder, for example, the function $|x|^{2}$ works for any $M$, and so uniqueness always holds.

For invariant equations on a sphere, existence always holds by Theorem 8.1.3. However, for domains which do not lie in a hemisphere, where there exists a convex function, comparison and its consequences can fail, even for pure second-order equations (see Appendix D in $\left.\left[\mathrm{HL}_{6}\right]\right)$.

- (Branches). The homogeneous Monge-Ampère equations over $\mathbf{R}, \mathbf{C}$ or $\mathbf{H}$ each have branches defined by $\lambda_{k}\left(D^{2} u\right)=0$ where $\lambda_{1} \leq \cdots \leq \lambda_{n}$ are the ordered eigenvalues. (See 2.1.3 and 2.1.10.) In fact the equation given by the $\ell^{\text {th }}$ elementary symmetric function $\sigma_{\ell}\left(D^{2} u\right)=0$ also has $\ell$ distinct branches. This is a general phenomenon which applies to any homogeneous polynomial on $\operatorname{Sym}^{2}\left(\mathbf{R}^{n}\right)$ 
which is Gårding hyperbolic with respect to the identity. (See $\left[\mathrm{HL}_{7,8}\right]$ and 4.3 .4 below.)

- (The Special Lagrangian Potential Equation). This equation $F(c)$, given in 2.2.1(d), can be treated for all values of $c$ and has the nice feature that $\widetilde{F}(c)=F(-c)$.

- (Geometrically Determined Subequations - Calibrations). These are subequations determined by a compact subset $\mathbb{G}$ of the Grassmann bundle of tangent $p$-planes by requiring that $\operatorname{tr}_{W}($ Hessu $u \geq 0$ for all $W \in \mathbb{G}$. These include many interesting examples, including the subequations in calibrated geometry discussed at the outset. It also includes a new polynomial differential equation in Lagrangian geometry (see 2.1.11(d)). Incidentally, this equation has branches whose study is a non-trivial application of the Gårding theory above.

- (Equations Involving the Principal Curvatures of the Graph and the $k$-Laplacian). For all such invariant equations on $G / H$, Theorem 8.1.3 gives existence (but not uniqueness). Strict boundary convexity is easily computable (see $\left[\mathrm{HL}_{6}, \S 17\right]$ for example). Existence holds on all domains for the $k$-Laplacian $|\nabla u|^{2} \Delta u+(k-2)(\nabla u)^{t}(\operatorname{Hess} u)(\nabla u)=0$, when $1<k \leq \infty$ and when $k=1$ on mean-convex domains, where uniqueness fails catastrophically.

A fundamental point is that all such equations can be carried over to any riemannian manifold with an appropriate (not necessarily integrable!) reduction of structure group. This is done by using the riemannian hessian given in $\S 8.2$. Theorem 8.1.2 can then be applied, and we obtain the following corollary. Let $\mathbf{F}$ and $\mathbf{M}$ be constant coefficient subequations in $\mathbf{R}^{n}$ with invariance group $G$.

TheOREM 8.2.2. Let $F$ be a subequation with monotonicity cone $M$ canonically determined by $\mathbf{F}$ and $\mathbf{M}$ on a riemannian manifold $X$ with a topological $G$-structure. Let $\Omega \subset \subset X$ be a domain with smooth boundary which is both $F$ and $\widetilde{F}$ srictly convex. Assume there exists a strictly $M$ subharmonic function on $\bar{\Omega}$. Then the Dirichlet Problem for F-harmonic functions is uniquely solvable for all $\varphi \in C(\partial \Omega)$.

- (Universal Riemannian Subequations). Any constant coefficient subequation $\mathbf{F}$ which in invariant under the natural action of $\mathrm{O}(n)$ carries over directly to any riemannian manifold, and Theorem 8.2.2 applies. This includes most of the examples above.

- (Universal Hermitian Subequations). A constant coefficient subequation $\mathbf{F}$ invariant under $\mathrm{U}(n)$ carries over to any almost complex hermitian manifold. There is a quaternionic analogue. More generally, we have:

- (Equations on Manifolds with $G$-Structure). A constant coefficient subequation $\mathbf{F}$ invariant under a subgroup $G \subset \mathrm{O}(n)$ carries 
over to any manifold equipped with a topological $G$-structure (see 8.2.1). This includes manifolds with topological (or quasi) calibrations based on any fixed form in $\Lambda^{p} \mathbf{R}^{n}$. Even the extreme case $G=\{e\}$ is interesting here. An $\{e\}$-structure is a topological trivialization of $T X$. It transplants every constant coefficient equation to $X$, and Theorem 8.2.2 applies. This holds, for example, for every orientable 3-manifold and every Lie group.

Theorem 8.1.2 actually treats much more general equations on manifolds. Affine jet-equivalence gives great flexibility to the result.

Many variable-coefficient, inhomogeneous subequations on manifolds can be transformed by local affine jet-equivalence to universally defined subequations, such as those in Theorem 8.2.2, while preserving the domains of strict boundary convexity.

- (Calabi-Yau-Type Equations). This is a good example of the power of affine jet equivalence. It applies to treat equations of type $(i \partial \bar{\partial} u+\omega)^{n}=F(x, u) \omega^{n}$ on almost complex hermitian manifolds, where $F>0$ is non-decreasing in $u$. See 3.2.8.

- (Inhomogeneous Equations). Many homogeneous equations can be transformed into inhomogeneous equations by affine jet equivalence. For example, from the $k^{\text {th }}$ branch of the MongeAmpère equation one can obtain: $\lambda_{k}(\operatorname{Hess} u)=f(x)$ for any continuous function $f$. See 3.2.7.

- (Obstacle Problems). The methods here apply also to the Dirichlet Problem with an Obstacle. In this case not all boundary data are allowed. They are constrained by the obstacle function. This is another example of an inhomogeneous equation. See $\S 8.6$.

- (Parabolic Equations). Each of these subequations has a parabolic cousin, where existence and uniqueness results are generally stronger. See 8.5.

For any subequation $F$ on a manifold $X$, one has the very natural

Restriction Question: When is the restriction of an $F$-subharmonic function on $X$ to a submanifold $j: Y \subset X$, a $j^{*}(F)$-subharmonic function on $Y$ ?

For $C^{2}$-functions, this always holds, and if fact defines the induced subequation $j^{*} F$. However, it is important and non-trivial for general upper semicontinuous subharmonics. There are several restriction results established in [HL9]. They are relevant to calibrated and riemannian geometry. Sometimes they lead to characterizing $F$-subharmonics in terms of their restrictions to special submanifolds.

An important case of this latter phenomenon occurs in almost complex manifolds. The "standard" way of defining plurisubharmonic functions is to require that the restrictions to (pseudo) holomorphic curves are subharmonic. There also exists an intrinsic subequation, whose subharmonics agree with the standard plurisubharmonic functions in the integrable case. Via the 
restriction theorem, these two definitions have been shown to agree on any almost complex manifold [ $\left.\mathrm{HL}_{10}\right]$.

There is also the notion of a plurisubharmonic distribution on a general almost complex manifold. Nefton Pali $[\mathrm{P}]$ has shown that those which are representable by continuous $[-\infty, \infty)$-valued functions are of the type above, and he conjectured that this should be true generally. This leads to another topic.

For convex subequations which are "second-order complete", a Strong Bellman Principle can be applied. It enables one to prove that distributionally $F$-subharmonic functions correspond in a very precise sense to the upper semi-continuous $F$-subharmonic functions considered here. This is done in $\left[\mathrm{HL}_{13}\right]$. Such arguments apply to prove the Pali Conjecture $\left[\mathrm{HL}_{10}\right]$.

Some Historical Notes. There is of course a vast literature on the principal branches of $\mathcal{P}$ and $\mathcal{P}^{\mathbf{C}}$ of the real and complex Monge-Ampère equations. Just to mention a few of the historically significant contributions beginning with Alexandrov: $[\mathrm{Al}],\left[\mathrm{Po}_{*}\right],[\mathrm{RT}],[\mathrm{B}],[\mathrm{W}],[\mathrm{TU}],\left[\mathrm{CNS}_{*}\right],[\mathrm{CKNS}]$, $\left[\mathrm{BT}_{*}\right],[\mathrm{HM}],\left[\mathrm{S}_{1}\right],\left[\mathrm{CY}_{*}\right]$, and [Yau]. Quaternionic subharmonicity and the principal branch $\mathcal{P}^{\mathbf{H}}$ of the quaternionic Monge-Ampère equation have been studied in $\left[\mathrm{A}_{*}\right]$ and $[\mathrm{AV}]$. On compact complex manifolds without boundary, viscosity solutions to equations of the form $(i \partial \bar{\partial} u+\omega)^{n}=e^{\varphi} v$, where $v>0$ is a given smooth volume form, were studied in [EGZ]. By establishing a comparison principle they obtain existence and uniqueness of solutions in important borderline cases $\left(\omega \geq 0, v \geq 0\right.$ with $\left.\int v>0\right)$, and also show that these are the unique solutions in the pluripotential sense.

The parabolic form of the 1-Laplacian gives rise to mean curvature flow by the level set method. Some of the interesting results on this topic (see $\left.\left[\mathrm{ES}_{*}\right],\left[\mathrm{CGG}_{*}\right],[\mathrm{E}],[\mathrm{Gi}]\right)$ can be carried over from euclidean space to the riemannian setting by the methods of $\left[\mathrm{HL}_{6}\right]$.

The first basic work on the Dirichlet Problem for the convex branches of the Special Lagrangian potential equation appeared in $\left[\mathrm{CNS}_{2}\right]$, and there are further results by Yuan $[\mathrm{Y}],[\mathrm{WY}]$.

In [AFS] and [PZ] standard viscosity theory has been adapted to riemannian manifolds by using the distance function, parallel translation, Jacobi fields, etc. For the problems considered here this machinery in not necessary.

In $\left[\mathrm{S}_{2,3,4}\right]$, Z. Slodkowski developed an axiomatic perspective on generalized subharmonic functions, and addressed the Dirichlet Problem in this context. He studied certain invariant "pseudoconvex classes" of functions on euclidean space and complex homogeneous spaces. There is a version of duality which plays an important role in his theory. It is formulated differently from the one here. However, in the cases of overlap the two notions of duality are equivalent. Interestingly, his results are used to prove a duality theorem for complex interpolation of normed spaces $\left[\mathrm{S}_{5}\right]$ 
Concerning Regularity. In this paper there is no serious discussion of regularity for solutions of the Dirichlet Problem. Indeed, with the level of degeneracy allowed here, no regularity above continuity can be claimed generally. Consider $u_{x x}=0$ in $\mathbf{R}^{2}$ for example. (See also [ $\left.\mathrm{Po}_{1}\right]$ and [NTV] and references therein.) A good account of regularity results can be found in [E]. A general exposition of viscosity methods and results appears in [CIL] and $[\mathrm{C}]$.

Concerning $-\infty$. Our approach here is to steadfastly treat subsolutions from the point of view of classical potential theory. We allow subsolutions ( $F$-subharmonic functions) to assume the value $-\infty$, in contrast to standard viscosity theory where subsolutions are finite-valued. This has the advantage of including basic functions, like the fundamental solution of the Laplacian, Riesz potentials, and $\log |f|$ with $f$ holomorphic, into the class of subsolutions. It also allows the constant function $u \equiv-\infty$, which is crucial for the restriction theorems discussed in Chapter 9. This issue is not important for the Dirichlet Problem.

\section{Subequations-A Geometric Approach}

The aim of this chapter is to present a geometric approach to subequations, pioneered by Krylov [K]. This point of view clarifies and conceptually simplifies many aspects of the theory. For transparency we begin with the basic case.

2.1. Constant Coefficient Subequations in $\mathbf{R}^{n}$. The 2-jets of functions on $\mathbf{R}^{n}$ (i.e., Taylor polynomials of degree two) take values in the vector space

$$
\mathbf{J}^{2} \equiv \mathbf{R} \times \mathbf{R}^{n} \times \operatorname{Sym}^{2}\left(\mathbf{R}^{n}\right) \quad \text { with traditional coordinates } \quad(r, p, A) .
$$

Definition 2.1.1. A second-order constant coefficient subequation on $\mathbf{R}^{n}$ is a proper closed subset $\mathbf{F} \subset \mathbf{J}^{2}$ satisfying the Positivity Condition

$$
\mathbf{F}+\mathcal{P} \subset \mathbf{F}
$$

and the Negativity Condition

$$
\mathbf{F}+\mathcal{N} \subset \mathbf{F}
$$

where

$$
\mathcal{P} \equiv\left\{(0,0, A) \in \mathbf{J}^{2}: A \geq 0\right\} \quad \text { and } \quad \mathcal{N} \equiv\left\{(r, 0,0) \in \mathbf{J}^{2}: r \leq 0\right\},
$$

and the Topological Condition

$$
\mathbf{F}=\overline{\operatorname{Int}} \mathbf{F} .
$$

We say $\mathbf{F}$ is pure second-order if $\mathbf{F}=\mathbf{R} \times \mathbf{R}^{n} \times \mathbf{F}_{0}$ for a closed subset $\mathbf{F}_{0} \subset \operatorname{Sym}^{2}\left(\mathbf{R}^{n}\right)$. In this case only $(\mathrm{P})$ is required, since $(\mathrm{N})$ is automatic and one can show that $(\mathrm{P}) \Rightarrow(\mathrm{T})$. Such subequations are often simply denoted by the subset $\mathbf{F}_{0}$ of $\operatorname{Sym}^{2}\left(\mathbf{R}^{n}\right)$. 
EXAMPLE 2.1.2. Some basic pure second-order examples are:

(a) The Laplace Subequation:

$$
\mathbf{F}_{0}=\left\{A \in \operatorname{Sym}^{2}\left(\mathbf{R}^{n}\right): \operatorname{tr} A \geq 0\right\} .
$$

(b) The Homogeneous Monge-Ampère Subequation:

$$
\mathbf{F}_{0}=\left\{A \in \operatorname{Sym}^{2}\left(\mathbf{R}^{n}\right): A \geq 0\right\} \cong \mathcal{P} .
$$

(c) The $k^{\text {th }}$ Elementary Symmetric Function Subequation:

$$
\mathbf{F}_{0}=\left\{A \in \operatorname{Sym}^{2}\left(\mathbf{R}^{n}\right): \sigma_{\ell}(A) \geq 0,1 \leq \ell \leq k\right\} .
$$

(d) The Special Lagrangian Potential Subequation:

$$
\mathbf{F}_{0}=\left\{A \in \operatorname{Sym}^{2}\left(\mathbf{R}^{n}\right): \operatorname{tr}(\arctan A) \geq c\right\} .
$$

(e) The Calabi-Yau Subequation: (This is not pure second-order, but it is gradient-independent.)

$$
\mathbf{F}=\left\{(r, p, A) \in \operatorname{Sym}^{2}\left(\mathbf{R}^{n}\right): \operatorname{tr}(A+I) \geq e^{r} \text { and } A+I \geq 0\right\} .
$$

REMARK 2.1.3. In $\mathbf{C}^{n}=\left(\mathbf{R}^{2 n}, J\right)$ each of the examples above has a complex analogue given by replacing $A$ with its hermitian symmetric part $A_{\mathbf{C}} \equiv \frac{1}{2}(A-J A J)$. The same applies in quaternionic $n$-space $\mathbf{H}^{n}=$ $\left(\mathbf{R}^{4 n}, I, J, K\right)$ with $A$ replaced by $A_{\mathbf{H}} \equiv \frac{1}{4}(A-I A I-J A J-K A K)$.

Definition 2.1.4. Given a constant coefficient subequation $\mathbf{F}$ on $\mathbf{R}^{n}$, the dual subequation $\widetilde{\mathbf{F}}$ is defined by

$$
\widetilde{\mathbf{F}} \equiv \sim(-\operatorname{Int} \mathbf{F})=-(\sim \operatorname{Int} \mathbf{F}) .
$$

LEMMA 2.1.5. F is a subequation $\Longleftrightarrow \widetilde{\mathbf{F}}$ is a subequation, and in this case

$$
\widetilde{\widetilde{\mathbf{F}}}=\mathbf{F} \quad \text { and } \quad \widetilde{\mathbf{F}+J}=\widetilde{\mathbf{F}}-J
$$

for all $J \in \mathbf{J}^{2}$.

The proof can be found in $\left[\mathrm{HL}_{4}, \S 4\right]$. In the examples above the dual subequations are easily computed in terms of the eigenvalues of $A$ (or $A_{\mathbf{C}}$, etc.). One finds that the Laplace subequation is self-dual $(\widetilde{\mathbf{F}}=\mathbf{F})$ but the others are generally not. Of particular interest is example (b) where the dual of $\mathcal{P} \equiv\{A \geq 0\}$ is

$$
\widetilde{\mathcal{P}} \cong\left\{A \in \operatorname{Sym}^{2}\left(\mathbf{R}^{n}\right): \text { at least one eigenvalue of } A \text { is } \geq 0\right\}
$$

We now present a concept of central importance which comes from viscosity theory [CIL]. For any manifold $X$, let $\operatorname{USC}(X)$ denote the set of upper semi-continuous functions $u: X \rightarrow[-\infty, \infty)$. Given $u \in \operatorname{USC}(X)$ and a point $x \in X$, a test function for $u$ at $x$ is a $C^{2}$-function $\varphi$ defined near $x$ so that

$$
u \leq \varphi \quad \text { and } \quad u(x)=\varphi(x)
$$


DeFinition 2.1.6. Let $\mathbf{F}$ be a constant coefficient subequation on $\mathbf{R}^{n}$ and fix an open set $X \subset \mathbf{R}^{n}$. A function $u \in \operatorname{USC}(X)$ is said to be $\mathbf{F}$ subharmonic on $X$ if for each $x \in X$ and each test function $\varphi$ for $u$ at $x$, the 2-jet (or total second derivative) of $\varphi$ satisfies

$$
J_{x}^{2} \varphi \equiv\left(\varphi(x),(D \varphi)_{x},\left(D^{2} \varphi\right)_{x}\right) \in \mathbf{F} .
$$

It is important that this condition (2.1.3) is only required at points where test functions actually exist. The set of such functions is denoted by $F(X)$.

It is striking that the space $F(X)$ of $F$-subharmonics shares many of the important properties enjoyed by classical subharmonic functions (see 2.3.1 below). The $C^{2}$-functions $u \in F(X)$ are exactly those with $J_{x}^{2} u \in \mathbf{F}$ for all $x \in X$. This basic fact requires the Positivity Condition $(\mathrm{P})$ on $\mathbf{F}$. Interestingly, the other properties in 2.3.1 do not require $(\mathrm{P})$.

For the subequation $\mathcal{P}$ in example (b) we have the following.

Proposition 2.1.7. (see [HL $\left.L_{4}, R m k .4 .9\right]$ and [HL $L_{9}$, Prop. 2.7])

(i) $\mathcal{P}(X)$ is the set of convex functions on $X$.

(ii) $\widetilde{\mathcal{P}}(X)$ is the set of subaffine functions on $X$.

Definition 2.1.8. A function $u \in \operatorname{USC}(X)$ is called subaffine if for each compact subset $K \subset X$ and each affine function $a$,

$$
u \leq a \text { on } \partial K \Rightarrow u \leq a \text { on } K .
$$

Note that subaffine functions satisfy the maximum principle. In fact, for a pure second-order subequations, the subequation $\widetilde{\mathcal{P}}$ is universal for this property. That is, if the functions in $\mathbf{F}(X)$ satisfy the maximum principle, then $\mathbf{F} \subset \widetilde{\mathcal{P}}$. We note also that functions which are locally subaffine are globally subaffine, while the corresponding statement for functions satisfying the maximum principle is false.

Definition 2.1.9. Let $\mathbf{F}$ and $X$ be as in Definition 2.1.6. A function $u \in \operatorname{USC}(X)$ is said to be $\mathbf{F}$-harmonic on $X$ if

$$
u \in F(X) \quad \text { and } \quad-u \in \widetilde{F}(X)
$$

Condition (2.1.4) implies that $u$ is continuous. If $u$ is twice differentiable at a point $x$, then (2.1.4) implies that

$$
J_{x}^{2} u \in \mathbf{F} \cap(-\widetilde{\mathbf{F}})=\mathbf{F} \cap(\sim \operatorname{Int} \mathbf{F})=\partial \mathbf{F} .
$$

Thus if $\mathbf{F}$ is defined classically as the closure of a set $\{f(r, p, A)>0\}$ for a continuous function $f: \mathbf{J}^{2} \rightarrow \mathbf{R}$, then any $u \in C^{2}(X)$ which is $\mathbf{F}$-harmonic satisfies the differential equation

$$
f\left(u, D u, D^{2} u\right)=0 \quad \text { on } X,
$$

however, the converse is not always true. 
Note 2.1.10. (Branches) It is instructive to consider the most basic of subequations, $\mathcal{P}$. A $C^{2}$-function $u$ which is $\mathcal{P}$-harmonic satisfies the homogeneous Monge-Ampère equation

$$
\operatorname{det}\left(D^{2} u\right)=0 \text {. }
$$

However, $u$ is required to have the additional property of being convex (cf. Alexandroff $[\mathrm{Al}]$ ). (In the complex analogue $u$ is plurisubharmonic.)

The equation (2.1.5) has other solutions corresponding to other "branches" of the locus $\{\operatorname{det} A=0\}$, which can also be handled by this theory. Given a symmetric matrix $A$, let $\lambda_{1}(A) \leq \lambda_{2}(A) \leq \cdots \leq \lambda_{n}(A)$ be the ordered eigenvalues of $A$. Since $\operatorname{det} A=\lambda_{1}(A) \cdots \lambda_{n}(A)$, equation (2.1.5) can be split into branches

$$
\lambda_{k}\left(D^{2} u\right)=0 .
$$

for $k=1, \ldots, n$. By monotonicity of eigenvalues, each $\boldsymbol{\Lambda}_{k} \equiv\left\{\lambda_{k} \geq 0\right\}$ is a subequation. Interestingly, the dual of a branch is another branch:

$$
\widetilde{\boldsymbol{\Lambda}_{k}}=\boldsymbol{\Lambda}_{n-k+1}
$$

This phenomenon of branches occurs in many equations of geometric significance.

Example 2.1.11. (Geometrically Defined Subequations) There is a large class of subequations which arise naturally in our set-theoretic setting. Let $G\left(p, \mathbf{R}^{n}\right)$ denote the Grassmannian of $p$-planes in $\mathbf{R}^{n}$. For each compact subset $\mathbb{G} \subset G\left(p, \mathbf{R}^{n}\right)$ we define the pure second-order subequation

$$
\mathbf{F}(\mathbb{G}) \equiv\left\{A \in \operatorname{Sym}^{2}\left(\mathbf{R}^{n}\right): \operatorname{tr}_{W} A \geq 0 \text { for all } W \in \mathbb{G}\right\}
$$

with dual

$$
\widetilde{\mathbf{F}(\mathbb{G})}=\left\{A \in \operatorname{Sym}^{2}\left(\mathbf{R}^{n}\right): \operatorname{tr}_{W} A \geq 0 \text { for some } W \in \mathbb{G}\right\}
$$

The $\mathbf{F}(\mathbb{G})$-subharmonic functions are called $\mathbb{G}$-plurisubharmonic. This terminology is justified by the following. Let $X \subset \mathbf{R}^{n}$ be an open set.

TheOrem 2.1.12. A function $u \in \operatorname{USC}(X)$ is $\mathbb{G}$-plurisubharmonic if and only if for every affine $\mathbb{G}$-plane $L$ the restriction $\left.u\right|_{X \cap L}$ is subharmonic for the standard Laplacian on L. The same statement holds with the affine $\mathbb{G}$-planes expanded to include all minimal $\mathbb{G}$-submanifolds of $X$. (A $\mathbb{G}$ submanifold is one whose tangent planes are elements of $\mathbb{G}$ ).

This follows from a Restriction Theorem in [HL9], which is discussed in Chapter 9.

(a) $\mathbb{G}=G\left(1, \mathbf{R}^{n}\right)$ : In this case $\mathbf{F}(\mathbb{G})=\mathcal{P}$ and the $\mathbb{G}$-plurisubharmonic functions are the classical convex functions, i.e., those which are convex on affine lines.

(b) $\mathbb{G}=G_{\mathbf{C}}\left(1, \mathbf{C}^{n}\right) \subset G\left(2, \mathbf{R}^{2 n}\right)$ the set of complex lines in $\mathbf{C}^{n}$ : In this case $\mathbf{F}(\mathbb{G})=\mathcal{P}^{\mathbf{C}}$ (see 4.3.1), and the $\mathbb{G}$-plurisubharmonic functions are the standard plurisubharmonic functions, i.e., those which are subharmonic on complex lines. 
(c) $\mathbb{G}=G\left(p, \mathbf{R}^{n}\right)$ : Here the $\mathbb{G}$-plurisubharmonic functions are the standard $p$-plurisubharmonic functions, i.e., those which are subharmonic on affine $p$-planes. This subequation has the feature that each $p$-plurisubharmonic function is also $\mathbb{G}$-plurisubharmonic for every closed $\mathbb{G} \subset G\left(p, \mathbf{R}^{n}\right)$. The analogue $\mathbb{G}=G\left(p, \mathbf{C}^{n}\right)$ in the complex case plays a role in analysis in several complex variables.

The $\mathbb{G}$-harmonic functions in these cases are viscosity solutions to differential equations which are $\mathrm{O}(n)$ (or $\mathrm{U}(n)$ ) invariant polynomials in the variables $D^{2} u$. Each of these equations has branches which will be discussed further in 4.3.1 and 4.3.2 below.

(d) $\mathbb{G}=$ LAG $\subset G\left(n, \mathbf{R}^{2 n}\right)$ the set of Lagrangian planes in $\mathbf{C}^{n}=\mathbf{R}^{2 n}$ : In this case the LAG-plurisubharmonic functions are relatively new and interesting. The corresponding harmonics are viscosity solutions to a differential equation which is a $\mathrm{U}(n)$-invariant polynomial in the variables $D^{2} u$ (see $\left[\mathrm{HL}_{14}\right]$ ). This equation also has branches.

Many important examples come directly from the theory of calibrations. A parallel calibration in $\mathbf{R}^{n}$ is a constant coefficient $p$-form whose restriction satisfies $\pm\left.\varphi\right|_{W} \leq \operatorname{vol}_{W}$ for all oriented $p$-planes $W$. For such a $\varphi$, we define $\mathbb{G} \equiv G(\varphi)$ to be the set of $W \in G\left(p, \mathbf{R}^{n}\right)$ such that $|\varphi|_{W} \mid=\operatorname{vol}_{W}$. In this case $G(\varphi)$ submanifolds (or simply $\varphi$-submanifolds) are automatically minimal. When $\varphi=\omega$ is the Kähler form in $\mathbf{C}^{n}$, we recover case (b) above, where the $\omega$-submanifolds are the holomorphic curves. (This carries over to any symplectic manifold $(X, \omega)$ with a compatible almost complex structure in the sense of Gromov [Gr].) The $G(\varphi)$ plurisubharmonic (or simply $\varphi$-plurisubharmonic) functions are essentially dual to the $\varphi$-submanifolds (see $\left[\mathrm{HL}_{2,3}\right]$ ), and they provide calibrated geometry with new tools from conventional analysis.

(e) $\mathbb{G}=G(\varphi)=\mathrm{SLAG} \subset G\left(n, \mathbf{R}^{2 n}\right)$ where $\varphi=\operatorname{Re}\left(d z_{1} \wedge \cdots \wedge d z_{n}\right)$ is the Special Lagrangian Calibration (cf. $\left[\mathrm{HL}_{1}\right]$ ). The notions of Special Lagrangian submanifolds and of SLAG-plurisubharmonic and SLAG-harmonic functions carry over to any Ricci-flat Kähler manifold (cf. $\left.\left[\mathrm{HL}_{1}\right]\right)$. The SLAG-subvarieties play a central role in the conjectured differential-geometric interpretation of mirror symmetry presented in $\left[\mathrm{SYZ}_{1,2}\right]$.

(f) $\mathbb{G}=G(\varphi) \subset G\left(3, \mathbf{R}^{7}\right)$ where $\mathbf{R}^{7}=\operatorname{Im} \mathbf{O}$ is the imaginary octonions and $\varphi(x, y, z) \equiv\langle x \cdot y, z\rangle$ is the associative calibration. There is a rich geometry of associative submanifolds, and an abundance of $\varphi$-plurisubharmonic and $\varphi$-harmonic functions. The same applies to the coassociative calibration $\psi=* \varphi$. Both calibrations make sense on any 7-manifold with $\mathrm{G}_{2}$-holonomy.

(g) $\mathbb{G}=G(\Phi) \subset G\left(4, \mathbf{R}^{8}\right)$ where $\mathbf{R}^{8}=\mathbf{O}$, the octonions, and $\Phi(x, y, z, w) \equiv\langle x \times y \times z, w\rangle$ is the Cayley calibration. There is a rich geometry of Cayley submanifolds, and an abundance of $\Phi$ plurisubharmonic and $\Phi$-harmonic functions. All this carries over to any 8-manifold with $\operatorname{Spin}_{7}$-holonomy. 
Note. While the $\varphi$-harmonic functions in examples (e), (f) and (g) are of basic interest in calibrated geometry, they appear not to satisfy any polynomial equation in $u, D u$ and $D^{2} u$. This is one justification for the approach to subequations adopted here.

2.2. Subequations on General Manifolds. Suppose now that $X$ is a smooth manifold of dimension $n$. The natural setting for second-order differential equations on $X$ is the bundle of 2-jets of functions on $X$. This is the bundle $J^{2}(X) \rightarrow X$ whose fibre at $x \in X$ is the quotient $J_{x}^{2}(X)=C_{x}^{\infty} / C_{x, 3}^{\infty}$ of germs of smooth functions at $x$ modulo those which vanish to order 3 at $x$.

Restriction from 2-jets to 1-jets gives a basic short exact sequence

$$
0 \longrightarrow \operatorname{Sym}^{2}\left(T^{*} X\right) \longrightarrow J^{2}(X) \longrightarrow J^{1}(X) \longrightarrow 0
$$

where $\operatorname{Sym}^{2}\left(T_{x}^{*} X\right)$ embeds into $J_{x}^{2}(X)$ as the 2-jets of functions having a critical value zero at $x$. The dual exact sequence is

$$
0 \longrightarrow J_{1}(X) \longrightarrow J_{2}(X) \stackrel{\sigma}{\longrightarrow} \operatorname{Sym}^{2}(T X) \longrightarrow 0 .
$$

Sections of $J_{k}(X)$ are linear differential operators of degree $\leq k$ on $X$, and $\sigma$ is the principal symbol map on operators of degree 2 .

There are two important, intrinsically defined subbundles of $J^{2}(X)$ which correspond to the subspaces $\mathcal{P}$ and $\mathcal{N}$ in Definition 2.1.1, namely:

$$
\begin{aligned}
\mathcal{P} & \equiv\left\{A \in \operatorname{Sym}^{2}\left(T^{*} X\right): A \geq 0\right\} \quad \text { and } \\
\mathcal{N} & \equiv\{2 \text {-jets of constant functions } \leq 0\} .
\end{aligned}
$$

Definition 2.2.1. A subequation of order $\leq 2$ on $X$ is a closed subset $F \subset J^{2}(X)$ satisfying (under fibre-wise sum) the Positivity Condition:

$$
F+\mathcal{P} \subset F,
$$

the Negativity Condition:

$$
F+\mathcal{N} \subset F
$$

and the Topological Condition:

(i) $F=\overline{\operatorname{Int} F}$,

(ii) $F_{x}=\overline{\operatorname{Int} F_{x}}$

(iii) $\operatorname{Int} F_{x}=(\operatorname{Int} F)_{x}$

where $\operatorname{Int} F_{x}$ denotes interior with respect to the fibre.

Note that $\mathcal{P}$ is not a subequation. However, when discussing pure secondorder subequations, it is sometimes used as an abbreviation for $\mathbf{R} \times \mathbf{R}^{n} \times \mathcal{P}$, which is a subequation. (see 2.1.1 and 2.1.2).

REMARK 2.2.2. (Splitting the 2-Jet Bundle) Let $\nabla$ be a torsion-free connection on $X$. Then each $u \in C^{2}(X)$ has an associated hessian Hess $u \in$ $\Gamma\left(\operatorname{Sym}^{2}\left(T^{*} X\right)\right)$ defined on vector fields $V, W$ by

$$
(\operatorname{Hess} u)(V, W)=V W u-W V u-\left(\nabla_{V} W\right) u .
$$


Since $\nabla_{V} W-\nabla_{W} V=[V, W]$, one easily sees that Hess $u$ is a symmetric tensor. If $X$ is riemannian and $\nabla$ is the Levi-Civita connection, then Hess $u$ is called the riemannian hessian of $u$.

The hessian in (2.2.3) depends only on the 2-jet of $u$ at each point, and so it gives a splitting of the short exact sequence (2.2.1). That is, we can write

$$
J^{2}(X)=\mathbf{R} \oplus T^{*} X \oplus \operatorname{Sym}^{2}\left(T^{*} X\right)
$$

by the association

$$
J_{x}^{2} u=\left(u(x),(d u)_{x}, \operatorname{Hess}_{x} u\right) .
$$

REMARK 2.2.3. (Universal Subequations) Each of the subequations given in Example 2.1.2 carries over to any riemannian manifold $X$ by using the splitting (2.2.4) (determined by the riemannian hessian). For instance, Example 2.1.2(a) gives the Laplace-Beltrami operator. More generally, any constant coefficient subequation $\mathbf{F} \subset \mathbf{J}^{2}$ which is invariant under the action of the group $\mathrm{O}(n)$, transplants to every riemannian manifold. In the case of $\mathbf{C}^{n}=\left(\mathbf{R}^{2 n}, J\right)$, each $\mathrm{U}(n)$-invariant subequation transplants to every hermitian almost complex manifold.

There is, in fact, a very general principle:

Let $\mathbf{F} \subset \mathbf{J}^{2}$ be a constant coefficient subequation which is invariant under a subgroup $G \subset O(n)$ acting naturally on $\mathbf{J}^{2}$. Then $\mathbf{F}$ carries over to a subequation $F$ on every manifold $X$ with a topological $G$-structure.

See $\left[\mathrm{HL}_{6}\right]$ and $\S 8.2$ below for definitions and many examples.

The concepts of the previous section carry over to this general setting.

Definition 2.2.4. Given a subequation $F \subset J^{2}(X)$, the dual subequation $\widetilde{F}$ is defined by

$$
\widetilde{F} \equiv \sim(-\operatorname{Int} F)=-(\sim \operatorname{Int} F)
$$

LEMMA 2.2.5.

$$
F \text { is a subequation } \Longleftrightarrow \widetilde{F} \text { is a subequation, }
$$

and in this case

$$
\widetilde{\widetilde{F}}=F \quad \text { and } \quad \widetilde{F+S}=\widetilde{F}-S
$$

for any section $S$ of $J^{2}(X)$.

The proof can be found in $\left[\mathrm{HL}_{6} \S 3\right]$. The dual of a universal subequation associated to $\mathbf{F} \subset \mathbf{J}^{2}$ is the universal subequation associated to $\widetilde{\mathbf{F}}$. As before we have the following.

Definition 2.2.6. Let $F$ be a subequation on a manifold $X$. A function $u \in \operatorname{USC}(X)$ is said to be $F$-subharmonic on $X$ if for each $x \in X$ and each test function $\varphi$ for $u$ at $x$,

$$
J_{x}^{2} \varphi \equiv\left(\varphi(x),(D \varphi)_{x},\left(D^{2} \varphi\right)_{x}\right) \in F .
$$

The set of such functions is denoted by $F(X)$. 
Definition 2.2.7. Let $F$ be a subequation on a manifold $X$. A function $u \in \operatorname{USC}(X)$ is said to be $F$-harmonic on $X$ if

$$
u \in F(X) \quad \text { and } \quad-u \in \widetilde{F}(X)
$$

As before, positivity ensures that a function $u \in C^{2}(X)$ is $F$-subharmonic on $X$ iff $J_{x}^{2} u \in F$ for all $x$, and it is $F$-harmonic iff

$$
J_{x}^{2} u \in \partial F \text { for all } x .
$$

2.3. Properties of F-Subharmonic Functions. The F-subharmonic functions share many of the important properties of classical subharmonic functions.

Theorem 2.3.1. (Elementary Properties of F-Subharmonic Functions) Let $F$ be an arbitrary closed subset of $J^{2}(X)$.

(i) (Maximum Property) If $u, v \in F(X)$, then $w=\max \{u, v\} \in F(X)$.

(ii) (Coherence Property) If $u \in F(X)$ is twice differentiable at $x \in X$, then $J_{x}^{2} u \in F_{x}$.

(iii) (Decreasing Sequence Property) If $\left\{u_{j}\right\}$ is a decreasing $\left(u_{j} \geq u_{j+1}\right)$ sequence of functions with all $u_{j} \in F(X)$, then the limit $u=$ $\lim _{j \rightarrow \infty} u_{j} \in F(X)$.

(iv) (Uniform Limit Property) Suppose $\left\{u_{j}\right\} \subset F(X)$ is a sequence which converges to $u$ uniformly on compact subsets to $X$, then $u \in F(X)$.

(v) (Families Locally Bounded Above) Suppose $\mathcal{F} \subset F(X)$ is a family of functions which are locally uniformly bounded above. Then the upper semicontinuous regularization $v^{*}$ of the upper envelope

$$
v(x)=\sup _{f \in \mathcal{F}} f(x)
$$

belongs to $F(X)$.

A proof can be found, for example, in Appendix B in $\left[\mathrm{HL}_{6}\right]$. For parts (i) and (ii), even the closure hypothesis on $F$ can be weakened (op. cit.).

\section{Jet Equivalence of Subequations}

Many important nonlinear equations that occur in geometry can be transformed locally to constant coefficient equations. This technique allows one to apply standard arguments from viscosity theory to prove local comparison results.

3.1. Affine Automorphisms of the Jet Bundle $J^{2}(X)$. The transformations we shall use are the affine automorphisms of $J^{2}(X)$ which we now introduce. To begin, note that there is a canonical direct sum decomposition

$$
J^{2}(X)=\mathbf{R} \oplus J_{\text {red }}^{2}(X)
$$


where the trivial $\mathbf{R}$-factor corresponds to the value of the function. For the reduced 2-jet bundle there is a short exact sequence

$$
0 \longrightarrow \operatorname{Sym}^{2}\left(T^{*} X\right) \longrightarrow J_{\text {red }}^{2}(X) \longrightarrow T^{*} X \longrightarrow 0
$$

coming from (2.2.1) above.

Definition 3.1.1. A linear isomorphism of $J^{2}(X)$ is an automorphism if, with respect to the splitting (3.1.1) it has the form $\operatorname{Id} \oplus \Phi$ where $\Phi: J_{\text {red }}^{2}(X) \rightarrow J_{\text {red }}^{2}(X)$ has the following properties. We first require that

$$
\Phi\left(\operatorname{Sym}^{2}\left(T^{*} X\right)\right)=\operatorname{Sym}^{2}\left(T^{*} X\right),
$$

so by (3.1.2) there is an induced bundle automorphism

$$
g=g_{\Phi}: T^{*} X \longrightarrow T^{*} X .
$$

We further require that there exist a second bundle automorphism

$$
h=h_{\Phi}: T^{*} X \longrightarrow T^{*} X
$$

such that on $\operatorname{Sym}^{2}\left(T^{*} X\right), \Phi$ has the form $\Phi(A)=h A h^{t}$, i.e.,

$$
\Phi(A)(v, w)=A\left(h^{t} v, h^{t} w\right) \quad \text { for } v, w \in T X .
$$

The automorphisms of $J^{2}(X)$ form a group. They are the sections of the bundle of groups $\operatorname{Aut}\left(J^{2}(X)\right)$ whose fibre at $x \in X$ is the group of automorphisms of $J_{x}^{2}(X)$ defined by (3.1.3) - (3.1.6) above. See $\left[\mathrm{HL}_{6}, \S 6.2\right]$ for this and the following.

Proposition 3.1.2. With respect to any splitting

$$
J^{2}(X)=\mathbf{R} \oplus T^{*} X \oplus \operatorname{Sym}^{2}\left(T^{*} X\right)
$$

of the short exact sequence (2.2.1), a bundle automorphism has the form

$$
\Phi(r, p, A)=\left(r, g p, h A h^{t}+L(p)\right)
$$

where $g, h: T^{*} X \rightarrow T^{*} X$ are bundle isomorphisms and $L$ is a smooth section of the bundle $\operatorname{Hom}\left(T^{*} X, \operatorname{Sym}^{2}\left(T^{*} X\right)\right)$.

Example 3.1.3. Given a local coordinate system $\left(\xi_{1}, \ldots, \xi_{n}\right)$ on an open set $U \subset X$, the canonical trivialization

$$
J^{2}(U)=U \times \mathbf{R} \times \mathbf{R}^{n} \times \operatorname{Sym}^{2}\left(\mathbf{R}^{n}\right)
$$

is determined by $J_{x}^{2} u=\left(u, D u, D^{2} u\right)$ where $D u=\left(u_{\xi_{1}}, \ldots, u_{\xi_{n}}\right)$ and $D^{2} u=$ $\left(\left(u_{\xi_{i} \xi_{j}}\right)\right)$ evaluated at the point $\xi(x) \in \mathbf{R}^{n}$. With respect to this splitting, every automorphism is of the form

$$
\Phi\left(u, D u, D^{2} u\right)=\left(u, g D u, h \cdot D^{2} u \cdot h^{t}+L(D u)\right)
$$

where $g_{x}, h_{x} \in \mathrm{GL}_{n}$ and $L_{x}: \mathbf{R}^{n} \rightarrow \operatorname{Sym}^{2}\left(\mathbf{R}^{n}\right)$ is linear for each point $x \in U$. 
EXAMPLE 3.1.4. The trivial 2-jet bundle on $\mathbf{R}^{n}$ has fibre

$$
\mathbf{J}^{2}=\mathbf{R} \times \mathbf{R}^{n} \times \operatorname{Sym}^{2}\left(\mathbf{R}^{n}\right) .
$$

with automorphism group

$$
\operatorname{Aut}\left(\mathbf{J}^{2}\right) \equiv \mathrm{GL}_{n} \times \mathrm{GL}_{n} \times \operatorname{Hom}\left(\mathbf{R}^{n}, \operatorname{Sym}^{2}\left(\mathbf{R}^{n}\right)\right)
$$

where the action is given by

$$
\Phi_{(g, h, L)}(r, p, A)=\left(r, g p, h A h^{t}+L(p)\right) .
$$

Note that the group law is

$$
(\bar{g}, \bar{h}, \bar{L}) \cdot(g, h, L)=\left(\bar{g} g, \bar{h} h, \bar{h} L \bar{h}^{t}+\bar{L} \circ g\right)
$$

Automorphisms at a point, with $g=h$, appear naturally when one considers the action of diffeomorphisms. Namely, if $\varphi$ is a diffeomorphism fixing a point $x_{0}$, then in local coordinates (as in Example 3.1.3 above) the right action on $J_{x_{0}}^{2}$, induced by the pull-back $\varphi^{*}$ on 2-jets, is an automorphism.

REMARK 3.1.5. Despite this last remark, automorphisms of the 2jet bundle $J^{2}(X)$, even those with $g=h$, have little to do with global diffeomorphisms or global changes of coordinates. In fact an automorphism radically restructures $J^{2}(X)$ in that the image of an integrable section (one obtained by taking $J^{2} u$ for a fixed smooth function $u$ on $X$ ) is essentially never integrable.

The automorphism group $\operatorname{Aut}\left(J^{2}(X)\right)$ can be naturally extended by the fibre-wise translations. Recall that the group of affine transformations of a vector space $V$ is the product $\operatorname{Aff}(V)=\mathrm{GL}(V) \times V$ acting on $V$ by $(g, v)(u)=g(u)+v$. The group law is $(g, v) \cdot(h, w)=(g h, v+g(w))$. There is a short exact sequence

$$
0 \rightarrow V \rightarrow \operatorname{Aff}(V) \stackrel{\pi}{\longrightarrow} \operatorname{GL}(V) \rightarrow\{I\} .
$$

Definition 3.1.6. The affine automorphism group of $J^{2}(X)$ is the space of smooth sections of

$$
\left.\pi^{-1}\left\{\operatorname{Aut}\left(J^{2}(X)\right)\right\}\right) \subset \operatorname{Aff}\left(J^{2}(X)\right)
$$

where $\pi$ is the surjective bundle map $\pi: \operatorname{Aff}\left(J^{2}(X)\right) \rightarrow \operatorname{GL}\left(J^{2}(X)\right)$.

Note that any affine automorphism can be written in the form

$$
\Psi=\Phi+S
$$

where $\Phi$ is a (linear) automorphism and $S$ is a section of the bundle $J^{2}(X)$. 


\subsection{Jet-Equivalence.}

Definition 3.2.1. Two subequations $F, F^{\prime} \subset J^{2}(X)$ are said to be jetequivalent if there exists an automorphism $\Phi: J^{2}(X) \rightarrow J^{2}(X)$ with $\Phi(F)=F^{\prime}$. If this holds for an affine automorphism $\Psi=\Phi+S$, they are said to be affinely jet-equivalent.

Remark 3.2.2. A jet-equivalence $\Phi: F \rightarrow F^{\prime}$ does not take $F$ subharmonic functions to $F^{\prime}$-subharmonic functions. In fact as mentioned above, for $u \in C^{2}, \Phi\left(J^{2} u\right)$ is almost never the 2-jet of a function. It happens if and only if $\Phi\left(J^{2} u\right)=J^{2} u$. Nevertheless, if $\Psi=\Phi+S$ is an affine automorphism of $J^{2}(X)$ and $F \subset J^{2}(X)$ is a closed set, then

$$
F \text { is a subequation } \quad \Longleftrightarrow \quad \Psi(F) \text { is a subequation, }
$$

and furthermore, by 2.2 .5 ,

$$
\widetilde{\Psi(F)}=\Phi(\widetilde{F})-S
$$

which is basic in establishing comparison.

Definition 3.2.3. We say that a subequation $F \subset J^{2}(X)$ is locally affinely jet-equivalent to a constant coefficient subequation $\mathbf{F}$ if each point $x$ has a local coordinate neighborhood $U$ such that, in the canonical trivialization (3.1.8) of $J^{2}(U)$ determined by those coordinates, $F$ is affinely jet-equivalent to the constant coefficient subequation $U \times \mathbf{F}$.

This concept is robust as shown by the following lemma, whose proof is a straightforward calculation.

LEMMA 3.2.4. If $F$ is affinely jet-equivalent to $\mathbf{F}$ in some local coordinate trivialization of $J^{2}(U)$, then this is true in every local coordinate trivialization of $J^{2}(U)$.

A basic reason for introducing this concept is the following (see $\left[\mathrm{HL}_{6}\right.$, Prop. 6.9]). Let $X$ be a riemannian manifold with topological $G$-structure for a subgroup $G \subset \mathrm{O}(n)$ (see (8.2.1)).

Proposition 3.2.5. Suppose that $F \subset J^{2}(X)$ is the subequation determined by a $G$-invariant constant coefficient subequation $\mathbf{F} \subset \mathbf{J}^{2}$ (cf. 2.2.3 and 8.2). Then $F$ is locally jet-equivalent to $\mathbf{F}$ on $X$.

EXAMPLE 3.2.6. (Universal Equations) Basic examples come from universal riemannian equations $(G=\mathrm{O}(n))$ such as those given in Example 2.1.2 (a), (b), (c), and their complex analogues on almost complex hermitian manifolds $(G=\mathrm{U}(n))$ or the analogues on almost quaternionic hermitian manifolds $(G=\operatorname{Sp}(n))$. There are also the other branches of these equations as discussed in Note 2.1.10. There are also the many geometric examples coming from Lagrangian geometry and calibrated geometry which are discussed below. 
ExAmple 3.2.7. (Inhomogeneous Equations) Another important fact about affine jet equivalence is that it can transform inhomogeneous equations into constant coefficient ones and vice versa. We present several illustrative examples here (and more in 8.5). They each have the structure $F=$ $\Psi(H), H=\Psi^{-1}(F)$ where $F$ is a pure second-order, universal riemannian subequation, and

$$
\Psi(A) \equiv h A h^{t}+S=\eta^{2} A+S
$$

where $h(x)=\eta(x) \mathrm{Id}$, for $\eta: X \rightarrow \mathbf{R}$, and $S: X \rightarrow \operatorname{Sym}^{2} T^{*}(X)$ is a translation term.

(i) Let $F$ correspond to the $k^{\text {th }}$ branch $\left\{\lambda_{k}(\right.$ Hess $\left.u)=0\right\}$ of the homogeneous Monge-Ampère equation (see 2.1.10). Taking $\eta \equiv 1$ and $S=-f(x)$ Id shows that $F$ is affinely jet-equivalent to the inhomogeneous equation

$$
\lambda_{k}(\operatorname{Hess} u)=f(x)
$$

for any smooth function $f$. This includes the Monge-Ampère equation from 2.1.2(b) when written as $\lambda_{\min }(\operatorname{Hess} u)=0$.

(ii) Let $F$ correspond to the universal equation $\operatorname{det}(\operatorname{Hess} u)=1$ with Hess $u \geq 0$. One can transform this to the inhomogeneous equation

$$
\operatorname{det}(\operatorname{Hess} u)=f(x) \quad \text { with } \quad \operatorname{Hess} u \geq 0
$$

for any smooth $f>0$ by choosing $\eta=f^{-\frac{1}{2 n}}$ and $S=0$.

(iii) More generally, one can transform the universal subequation: $\sigma_{k}(\operatorname{Hess} u)=1$ and $\sigma_{\ell}(\operatorname{Hess} u) \geq 0, \quad 1 \leq \ell<k$, into the inhomogeneous equation

$\sigma_{k}(\operatorname{Hess} u)=f(x) \quad$ and $\quad \sigma_{\ell}(\operatorname{Hess} u) \geq 0, \quad 1 \leq \ell<k$

for any smooth $f>0$ by choosing $\eta=f^{-\frac{1}{2 k}}$ and $S=0$.

Example 3.2.8. (The Calabi-Yau Equation) Let $X$ be an almost complex hermitian manifold (a Riemannian $\mathrm{U}_{n}$-manifold), and consider the subequation $F \subset J^{2}(X)$ determined by the euclidean subequation:

$$
\operatorname{det}_{\mathbf{C}}\left\{A_{\mathbf{C}}+I\right\} \geq 1 \text { and } A_{\mathbf{C}}+I \geq 0
$$

where $A_{\mathbf{C}} \equiv \frac{1}{2}(A-J A J)$ is the hermitian symmetric part of $A$. Let $f>0$ be a smooth positive function on $X$ and write $f=h^{-2 n}$. Consider the global affine automorphism of $J^{2}(X)$ given by

$$
\Psi(r, p, A)=\left(r, p, h^{2} A+\left(h^{2}-1\right) I\right)
$$

and set $F_{f}=\Psi^{-1}(F)$. Then

$$
\begin{aligned}
(r, p, A) \in F_{f} & \Longleftrightarrow \quad \operatorname{det}_{\mathbf{C}}\left\{h^{2}\left(A_{\mathbf{C}}+I\right)\right\} \geq 1 \text { and } h^{2}\left(A_{\mathbf{C}}+I\right) \geq 0 \\
& \Longleftrightarrow \quad \operatorname{det}_{\mathbf{C}}\left\{\left(A_{\mathbf{C}}+I\right)\right\} \geq f \text { and }\left(A_{\mathbf{C}}+I\right) \geq 0
\end{aligned}
$$

so we see that the $F_{f}$-harmonic functions are functions $u$ with $\operatorname{det}_{\mathbf{C}}\left\{\operatorname{Hess}_{\mathbf{C}} u+I\right\}=f$ and $\operatorname{Hess}_{\mathbf{C}} u+I \geq 0$ (quasi-plurisubharmonic). 
If $X$ is actually a complex manifold of dimension $n$ with Kähler form $\omega$, this last equation can be written in the more familiar form

$$
(i \partial \bar{\partial} u+\omega)^{n}=f \omega^{n}
$$

with $u$ quasi-plurisubharmonic.

One can similarly treat the equation

$$
(i \partial \bar{\partial} u+\omega)^{n}=e^{u} f \omega^{n} .
$$

or the same equation with $e^{u}$ replaced by any non-decreasing positive function $F(u)$.

The concept of affine jet equivalence plays a critical role in the study of intrinsically subharmonic functions on almost complex manifolds $\left[\mathrm{HL}_{10}\right]$.

\section{Monotonicity.}

A concept of fundamental importance here is that of a monotonicity cone for a given subequation. It is the key to establishing comparison and removable singularity theorems for equations which are highly non-convex.

4.1. The Constant Coefficient Case. Let $\mathbf{F}, \mathbf{M} \subset \mathbf{J}^{2}$ be constant coefficient subequations.

Definition 4.1.1. We say that $\mathbf{M}$ is a monotonicity subequation for $\mathbf{F}$ if

$$
\mathbf{F}+\mathbf{M} \subset \mathbf{F} .
$$

It follows directly from 2.1.6 that the sum of an $\mathbf{F}$-subharmonic function and an $\mathbf{M}$-subharmonic function is again $\mathbf{F}$-subharmonic, provided that one of them is smooth. Thus, the reader can see that monotonicity is related to approximation whenever $\mathbf{M}$ has the cone property

$$
t \mathbf{M} \subset \mathbf{M} \quad \text { for } 0 \leq t \leq 1 .
$$

When this holds $M$ can be expanded so that each fibre is a convex cone with vertex at the origin (cf. 4.1.4). Under this added assumption $\mathbf{M}$ is called a monotonicity cone.

LEMMA 4.1.2. If $\mathbf{M}$ is a monotonicity cone for $\mathbf{F}$, then

$$
\begin{aligned}
& \widetilde{\mathbf{F}}+\mathbf{M} \subset \widetilde{\mathbf{F}} \quad \text { and } \\
& \mathbf{F}+\widetilde{\mathbf{F}} \subset \widetilde{\mathbf{M}} .
\end{aligned}
$$

These elementary facts are basic. The first states that:

$\mathbf{M}$ is a monotonicity cone for $\mathbf{F} \Longleftrightarrow \mathbf{M}$ is a monotonicity cone for $\widetilde{\mathbf{F}}$.

The second is the algebraic precursor to proving that:

The sum of an $\mathbf{F}$-subharmonic function and an $\widetilde{\mathbf{F}}$-subharmonic function is $\widetilde{\mathbf{M}}$-subharmonic. 
If one of the two functions is smooth, this last result follows easily from the definitions. It is important, because in most cases, the $\widetilde{\mathbf{M}}$-subharmonic functions satisfy the following:

Zero Maximum Principle: For any compact set $K$ in the domain of $u$,

$$
u \leq 0 \text { on } \partial K \Rightarrow u \leq 0 \text { on } K
$$

Example 4.1.3. The (ZMP) holds for $\widetilde{\mathbf{M}}$-subharmonic functions when

$$
\mathbf{M}=\left\{(r, p, A) \in \mathbf{J}^{2}: r \leq-\gamma|p|, \quad p \in \mathcal{D} \text { and } A \geq 0\right\}
$$

where $\gamma>0$ and $\mathcal{D} \subset \mathbf{R}^{n}$ is a convex cone with non-empty interior (and vertex at 0). See Appendix B for a proof and further discussion of Examples. Note incidentally that the smaller $M$ is, the easier it is to be a monotonicity cone for $F$, while the larger $\widetilde{M}$ is, the harder it is to satisfy (ZMP).

Note 4.1.4. Associated to any subequation $\mathbf{F}$ is the set $\mathbf{M}_{\mathbf{F}}$ of all $J \in \mathbf{J}^{2}$ such that $\mathbf{F}+t J \subset \mathbf{F}$ for $0 \leq t \leq 1$. One checks easily that $\mathbf{M}_{\mathbf{F}}$ is a closed convex cone which satisfies $(\mathrm{P})$ and $(\mathrm{N})$. Thus, if $\operatorname{Int}_{\mathbf{F}} \neq \emptyset$, it is the maximal monotonicity cone for $\mathbf{F}$.

4.2. The General Case. Let $F \subset J^{2}(X)$ be a subequation on a manifold $X$.

Definition 4.2.1. A monotonicity cone for $F$ is a convex cone subequation $M \subset J^{2}(X)$ (each fibre is a convex cone with vertex at the origin) satisfying the condition

$$
F+M \subset F
$$

Lemma 4.2.2. If $M$ is a monotonicity cone for $F$, then

$$
\begin{aligned}
& \widetilde{F}+M \subset \widetilde{F} \quad \text { and } \\
& F+\widetilde{F} \subset \widetilde{M} .
\end{aligned}
$$

Note 4.2.3. Suppose $\mathbf{F} \subset \mathbf{J}^{2}$ is a constant coefficient subequation invariant under a subgroup $G \subset \mathrm{O}(n)$. Then $\mathbf{M}_{\mathbf{F}}$ is also $G$-invariant. Thus if $\operatorname{Int} \mathbf{M}_{\mathbf{F}} \neq \emptyset$, it determines a monotonicity cone $M_{F}$ for every subequation $F$ canonically determined on any manifold with a topological $G$-structure (cf. Remark 2.2.3).

4.3. Examples. (Branches of Polynomial Equations) Many subequations have naturally associated monotonicity cones. The most basic case is the following.

Example 4.3.1. (Homogeneous Monge Ampère Equations) Let $K=$ $\mathbf{R}, \mathbf{C}$ or $\mathbf{H}$ and let $K^{n}=\mathbf{R}^{N}$ for $N / n=1,2$, or 4 . Then any quadratic form $A \in \operatorname{Sym}^{2}\left(\mathbf{R}^{N}\right)$ has a $K$-hermitian symmetric part $A_{K}$ defined in Remark 2.1.3. Let $\lambda_{1}^{K}(A) \leq \cdots \leq \lambda_{n}^{K}(A)$ be the ordered eigenvalues of $A_{K}$ 
(where we ignore the natural multiplicities 2 in the complex case and 4 in the quaternion case). Let

$$
\Lambda_{k}^{K} \equiv\left\{\lambda_{k}^{K}(A) \geq 0\right\}
$$

denote the $k^{\text {th }}$ branch of the homogeneous Monge-Ampère equation (cf. Note 2.1.10). The dual subequation is $\widetilde{\Lambda}_{k}^{K}=\Lambda_{n-k+1}^{K}$. These subequations carry over to any riemannian manifold with orthogonal almost complex or quaternionic structures.

The smallest, most basic branch is $\boldsymbol{\Lambda}_{1}^{K}=\left\{A^{K} \geq 0\right\}=\mathbf{F}\left(G\left(1, K^{n}\right)\right)$, which will be denoted by $\mathcal{P}^{K}, K=\mathbf{R}, \mathbf{C}$ or $\mathbf{H}$. The monotonicity of ordered eigenvalues: $\lambda_{k}^{K}(A) \leq \lambda_{k}^{K}(A+P)$ for $P \in \mathcal{P}^{K}$ implies that

$$
\boldsymbol{\Lambda}_{k}^{K}+\mathcal{P}^{K} \subset \boldsymbol{\Lambda}_{k}^{K},
$$

i.e., the top branch $\mathcal{P}^{K}$ is a monotonicity cone for each branch $\boldsymbol{\Lambda}_{k}^{K}$ of the Monge-Ampère equation.

Example 4.3.2. (p-Convexity) Fix $p, 1 \leq p \leq n$. For each $A \in$ $\operatorname{Sym}^{2}\left(\mathbf{R}^{n}\right)$ and each $p$-tuple $I=\left\{i_{1}<i_{2}<\cdots<i_{p}\right\}$, set $\lambda_{I}(A)=$ $\lambda_{i_{1}}(A)+\cdots+\lambda_{i_{p}}(A)$. Consider the second-order polynomial differential equation determined by

$$
\mathrm{MA}_{p}(A) \equiv \prod_{I} \lambda_{I}(A)=\operatorname{det}\left\{D_{A}: \Lambda^{p} \mathbf{R}^{n} \rightarrow \Lambda^{p} \mathbf{R}^{n}\right\}=0
$$

where $D_{A}$ denotes $A$ acting as a derivation on the exterior power $\Lambda^{p} \mathbf{R}^{n}$. This equation splits into branches $\boldsymbol{\Lambda}_{k}(p), k=1, \ldots,\left(\begin{array}{l}n \\ p\end{array}\right)$, obtained by ordering the eigenvalues $\left\{\lambda_{I}(A)\right\}$. The principle branch $\boldsymbol{\Lambda}_{1}(p)$, which is denoted by

$$
\mathcal{P}(p) \equiv\left\{A: \lambda_{1}(A)+\cdots+\lambda_{p}(A) \geq 0\right\}=\mathbf{F}\left(G\left(p, \mathbf{R}^{n}\right)\right),
$$

is exactly the one considered in 2.1.11(c). In particular, the $\mathcal{P}(p)$-subharmonic functions are just the $p$-plurisubharmonic functions - those which are harmonic on all affine p-planes. The monotonicity of eigenvalues shows that $\mathcal{P}(p)$ is a monotonicity cone for every branch of this equation, that is,

$$
\boldsymbol{\Lambda}_{k}(p)+\mathcal{P}(p) \subset \boldsymbol{\Lambda}_{k}(p) .
$$

More generally, let $K=\mathbf{R}, \mathbf{C}$ or $\mathbf{H}$ and, using the notation of 4.3.1, set

$$
\operatorname{MA}_{p}^{K}(A) \equiv \prod_{I} \lambda_{I}^{K}(A)
$$

This defines a polynomial differential equation with principal branch $\mathcal{P}^{K}(p)=\mathbf{F}\left(G\left(p, K^{n}\right)\right)$. The other branches, obtained as above by ordering the eigenvalues $\left\{\lambda_{I}^{K}(A)\right\}$, are subequations for which $\mathcal{P}^{K}(p)$ is a monotonicity cone.

The cone $\mathcal{P}(p)$ can be defined for any real number $p, 1 \leq p \leq n$ by

$$
\mathcal{P}(p) \equiv\left\{A: \lambda_{1}(A)+\cdots+\lambda_{[p]}(A)+(p-[p]) \lambda_{p+1}(A) \geq 0\right\} .
$$

This extension plays an important role in removable singularity theorems (see Section 6.2 below). We note that this extended $\mathcal{P}(p)$ is the principal 
branch of the polynomial operator $\mathrm{MA}_{p}(A)=\prod\left(\lambda_{I}(A)+(p-[p]) \lambda_{k}(A)\right)$ where the product is over $|I|=[p]-1$ and $k \notin I$.

EXAMPLE 4.3.3. ( $\delta$-Uniform Ellipticity) A basic family of monotonicity subequations is given by

$$
\mathcal{P}(\delta) \equiv\left\{A \in \operatorname{Sym}^{2}\left(\mathbf{R}^{n}\right): A \geq-\delta \operatorname{tr} A \cdot I\right\}
$$

for $\delta>0$. Any subequation $\mathbf{F}$, for which $\mathcal{P}(\delta)$ is a monotonicity cone, is uniformly elliptic in the usual sense. This subequation is the principal branch of the pure second-order polynomial differential equation:

$$
\prod_{i=1}^{n}\left(\lambda_{k}(\operatorname{Hess} u)+\delta \Delta u\right)=0 .
$$

This equation has $n$ branches

$$
\lambda_{k}(\operatorname{Hess} u)+\delta \Delta u \geq 0 \quad \text { for } k=1, \ldots, n,
$$

and $\mathcal{P}(\delta)$ is a monotonicity cone for each of these branches, so in particular, each branch is uniformly elliptic.

This is easily generalized as follows. Suppose $\mathbf{F} \subset \operatorname{Sym}^{2}\left(\mathbf{R}^{n}\right)$ is any pure second-order subequation. Then for each $\delta>0$, the $\delta$-elliptic regularization $\mathbf{F}(\delta)$ is defined by requiring that $A+\delta(\operatorname{tr} A) \cdot I \in \mathbf{F}$. Now if $\mathbf{M}$ is a monotonicity cone for $\mathbf{F}$, it follows immediately from the definitions that $\mathbf{M}(\delta)$ is a monotonicity cone for $\mathbf{F}(\delta)$. Also, $\mathcal{P} \subset \mathbf{M}$ implies that $\mathcal{P}(\delta) \subset$ $\mathbf{M}(\delta)$, which ensures that each $\mathbf{F}(\delta)$ is uniformly elliptic.

Example 4.3.4. Gårding Hyperbolic Polynomials) The examples above, and several below, fall into a general class of equations where monotonicity cones appear naturally. A homogeneous polynomial $Q: \operatorname{Sym}^{2}\left(\mathbf{R}^{n}\right) \rightarrow \mathbf{R}$ of degree $m$ is said to be Gårding hyperbolic with respect to the identity if $Q(I)=1$ and for each $A \in \operatorname{Sym}^{2}\left(\mathbf{R}^{n}\right)$ the polynomial $q_{A}(t) \equiv Q(t I+A)$ has $m$ real roots. Thus we can write

$$
Q(t I+A)=\prod_{k=1}^{m}\left(t+\lambda_{k}(A)\right)
$$

where the $\lambda_{1}(A) \leq \cdots \leq \lambda_{m}(A)$ are the ordered eigenvalues (the negatives of the roots) of $q_{A}(t)$. Such a polynomial has $m$ branches

$$
\boldsymbol{\Lambda}_{Q, k} \equiv\left\{\lambda_{k}(A) \geq 0\right\}, \quad k=1, \ldots, m,
$$

which correspond to $m$ constant coefficient pure second-order subequations in $\mathbf{R}^{n}$. The principal branch

$$
\mathbf{M}_{Q} \equiv \boldsymbol{\Lambda}_{Q, 1}
$$

is called the Gårding cone. Gårding's beautiful theory of hyperbolic polynomials $[\mathrm{G}]$ applies here to give the following. 
Proposition 4.3.5. The Garding cone $\mathbf{M}_{Q}$ is a convex cone containing the identity I. It satisfies the property

$$
\boldsymbol{\Lambda}_{Q, k}+\mathbf{M}_{Q} \subset \boldsymbol{\Lambda}_{Q, k} \text { for all } k=1, \ldots, m,
$$

that is, $\mathbf{M}_{Q}$ gives a monotonicity cone for each of the subequations $\boldsymbol{\Lambda}_{Q, k}$. In particular, as long as $\mathbf{M}_{Q}$ contains $\mathcal{P}$, each branch $\Lambda_{Q, k}$ of $Q$ is a subequation.

One of the simplest examples comes by taking $Q(A)=\sigma_{m}(A)$, the $m^{\text {th }}$ elementary symmetric function in the eigenvalues. Here the Garding cone $\mathbf{M}_{Q}$ is the set $\left\{\sigma_{1} \geq 0, \ldots, \sigma_{m} \geq 0\right\}$ (cf. Example 2.1.2(c)).

In general, for any hyperbolic polynomial $Q$ as above, one can construct large families of associated subequations, equipped with monotonicity cones, by using the eigenvalues of $Q$. For a discussion of this as well as an elementary introduction to Gårding's theory, see [HL $\left.\mathrm{HL}_{7,8}\right]$.

4.4. Monotonicity and Duality. The key algebraic fact that the dual of a translated subequation $F+J$ is just $\widetilde{F}-J$ (see 2.1 .5 ) easily proves the following result, which in turn proves the basic algebraic lemmas 4.1 .2 and 4.2.2.

Lemma 4.4.1. Given three subequations $G, M, F \subset J^{2}(X)$, the fibre-wise sums satisfy:

$$
G+M \subset F \quad \Longleftrightarrow \quad G+\widetilde{F} \subset \widetilde{M} .
$$

Proof. Note that $J+M \subset F \Longleftrightarrow M \subset-J+F \Longleftrightarrow J+\widetilde{F} \subset \widetilde{M}$.

Later on, (4.4.1) will be implemented with $G=F^{c} \subset F$ (cf. (5.1.1)) to obtain weak comparison (see Remark 5.1.4).

4.5. Uniform Ellipticity as Monotonicity. As noted in Example 4.4.3 the classical notion of uniform ellipticity can be reformulated in terms of monotonicity. We now examine this in greater detail. Suppose that $F$ is a subequation defined on an open set $X \subset \mathbf{R}^{n}$, in the classical way, by $F \equiv\{f(x, r, p, A) \geq 0\}$ for a function $f: J^{2}(X) \rightarrow \mathbf{R}$ (cf. Appendix A). Then uniform ellipticity (with constants $0<\lambda<\Lambda$ ) is the condition that for $A, P \in \operatorname{Sym}^{2}\left(\mathbf{R}^{n}\right)$ with $P \geq 0$,

$$
\lambda \operatorname{tr}(P) \leq f(x, r, p, A+P)-f(x, r, p, A) \leq \Lambda \operatorname{tr}(P)
$$

(and is usually combined with Lipschitz continuity in $p$ ). This condition can be reformulated in terms of a monotonicity subequation for $F$. To see this it suffices to consider the simplest case $f: \operatorname{Sym}^{2}\left(\mathbf{R}^{n}\right) \rightarrow \mathbf{R}$. The condition (4.5.1) is equivalent to requiring that for all $A, B$ (not just $B \geq 0$ ),

$$
\mathcal{P}_{\lambda, \Lambda}^{-}(B) \leq f(A+B)-f(A) \leq \mathcal{P}_{\lambda, \Lambda}^{+}(B)
$$

where $\mathcal{P}_{\lambda, \Lambda}^{ \pm}$are the Pucci operators defined by

$$
\mathcal{P}_{\lambda, \Lambda}^{-}(B) \equiv \lambda \operatorname{tr}\left(B^{+}\right)+\Lambda \operatorname{tr}\left(B^{-}\right) \quad \text { and } \quad \mathcal{P}_{\lambda, \Lambda}^{+}(B) \equiv-\mathcal{P}_{\lambda, \Lambda}^{-}(-B)
$$


and where $B=B^{+}+B^{-}$is the decomposition into $B^{+} \geq 0$ and $B^{-} \leq 0$. It is easy to see that the left hand inequality in $(4.5 .1)^{\prime}$ for all $A, B$ is equivalent to the right hand inequality for all $A, B$. The desired monotonicity is given by the Pucci cone

$$
\mathbf{P}_{\lambda, \Lambda} \equiv\left\{B \in \operatorname{Sym}^{2}\left(\mathbf{R}^{n}\right): \mathcal{P}_{\lambda, \Lambda}^{-}(B) \geq 0\right\} .
$$

Note that the left hand inequality in (4.5.1)' implies the monotonicity:

$$
F+\mathbf{P}_{\lambda, \Lambda} \subset F \text {. }
$$

The equivalence of $F+\mathbf{P}_{\lambda, \Lambda} \subset F$ and $\widetilde{F}+\mathbf{P}_{\lambda, \Lambda} \subset \widetilde{F}$ corresponds to the equivalence of the right and left hand inequalities in (4.5.1)'.

The Pucci cones are convex. One way to see this is to compute that $\mathbf{P}_{\lambda, \Lambda}$ is the polar of the convex cone on the set $\left\{B \in \operatorname{Sym}^{2}\left(\mathbf{R}^{n}\right): \lambda I \leq B \leq \Lambda I\right\}$.

We point out that Pucci cones provide just one of many choices of a family of monotonicity subequations (convex cones) which form a "fundamental" neighborhood system of $\mathcal{P}=\{A \geq 0\}$, e.g. Example 4.3.3 above. All such families give equivalent notions of uniform ellipticity.

\section{Comparison and Strict Approximation}

Let $F \subset J^{2}(X)$ be a subequation on a manifold $X$ and for each compact set $K \subset X$ set $F(K)=\operatorname{USC}(K) \cap F(\operatorname{Int} K)$.

Definition 5.0.1. We say that comparison holds for $F$ on $X$ if for every compact subset $K$, the Zero Maximum Principle

$$
u+v \leq 0 \text { on } \partial K \Rightarrow u+v \leq 0 \text { on } K
$$

holds for all

$$
u \in F(K) \quad \text { and } \quad v \in \widetilde{F}(K) .
$$

One sees easily that comparison implies uniqueness for the Dirichlet problem:

If $u$ and $v$ are $F$-harmonic on $\operatorname{Int} K$ and $u=v$ on $\partial K$, then $u=v$ on $K$

5.1. Weak Comparison. A $C^{2}$ function $u$ on $X$ is said to be strictly $F$-subharmonic if $J_{x}^{2} u \in \operatorname{Int} F_{x}$ for all $x$. This notion has the following useful extension to functions which are not $C^{2}$. For $c>0$ let $F^{c}$ be the subequation with fibres

$$
F_{x}^{c} \equiv\left\{J \in F_{x}: \operatorname{dist}\left(J, \sim F_{x}\right) \geq c\right\}
$$

where dist denotes distance in the fibre $J_{x}^{2}(X)$. This set satisfies conditions $(\mathrm{P})$ and $(\mathrm{N})$. A function $u \in \operatorname{USC}(X)$ is called strictly $F$-subharmonic if each $x$ has a neighborhood $U$ and $c>0$ such that $u$ is $F^{c}$-subharmonic on $U$.

Definition 5.1.1. We say that weak comparison holds for $F$ on $X$ if for every compact subset $K$,

$$
u+v \leq 0 \text { on } \partial K \Rightarrow u+v \leq 0 \text { on } K
$$


holds for all

$$
u \in F^{c}(K), \quad v \in \widetilde{F}(K) \text { and } c>0 .
$$

We say that local weak comparison holds for $F$ on $X$ if every point has a neighborhood in which weak comparison holds. This weakened form of comparison has several advantages. The first is the following.

THEOREM 5.1.2. (Local implies Global) If local weak comparison holds on $X$, then weak comparison holds on $X$.

A second important advantage is the following.

TheOREM 5.1.3. Suppose $F$ is a subequation on $X$ which is locally jetequivalent to a constant coefficient subequation. Then local weak comparison holds for $F$ on $X$.

REMARK 5.1.4. $F^{c}$ is exactly the subset of $F$ which satisfies the "weak monotonicity"

$$
F^{c}+M^{c} \subset F \quad \text { and hence } \quad F^{c}+\widetilde{F} \subset \widetilde{M^{c}}
$$

where $M^{c}$ is the universal subequation corresponding to the constant coefficient subequation

$$
\mathbf{M}^{c} \equiv(-\infty, 0] \times \overline{B(0, c)} \times(\mathcal{P}-c \cdot I) .
$$

The smaller subequation $\mathbf{M}_{c} \subset \mathbf{M}^{c}$ defined by

$$
\mathbf{M}_{c} \equiv(-\infty, 0] \times \overline{B(0, c)} \times \mathcal{P}
$$

has dual $\widetilde{\mathbf{M}}_{c} \supset \widetilde{\mathbf{M}}^{c}$ which satisfies the (ZMP). It is the union of three subequations:

$$
\begin{gathered}
\mathbf{R}_{-} \times \mathbf{R}^{n} \times \operatorname{Sym}^{2}\left(\mathbf{R}^{n}\right) \quad \text { (zeroth order) } \\
\mathbf{R} \times(\sim B(0, c)) \times \operatorname{Sym}^{2}\left(\mathbf{R}^{n}\right) \quad \text { (dual Eikonal) } \\
\mathbf{R} \times \mathbf{R}^{n} \times \widetilde{\mathcal{P}} \quad \text { (subaffine) },
\end{gathered}
$$

5.2. Strict Approximation. We say that strict approximation holds for $F$ on $X$ if for each compact set $K \subset X$, each function $u \in F(K)$ can be uniformly approximated by functions in $F(K)$ which are strict on Int $K$.

THEOREM 5.2.1. If weak comparison and strict approximation hold for $F$ on $X$, then comparison holds for $F$ on $X$.

TheOREM 5.2.2. Let $F$ be a subequation on $X$ with a monotonicity cone subequation $M$. Suppose $X$ carries a $C^{2}$-function which is strictly $M$ subharmonic. Then local weak comparison implies global comparison for $F$ on $X$.

The idea is to approximate $u \in F(K)$ by $u+\epsilon \psi, \epsilon>0$, where $\psi$ is the strictly $M$-subharmonic function. (The proofs of these theorems can be found in $\left[\mathrm{HL}_{6}\right]$.) 
Thus we see that monotonicity subequations are of central importance in solving the Dirichlet Problem for nonlinear equations which are degenerate and highly non-convex.

There are times when strict approximation can be achieved by other means. One example is given by the Eikonal subequation $|\nabla u| \leq 1$. Here the family of functions $u_{\epsilon}=(1-\epsilon) u$ for $\epsilon>0$ gives strict approximation.

5.3. Addition Theorems. In $\left[\mathrm{HL}_{4}\right]$ the following results were proved for pure second-order, constant coefficient subequations on an open subset $X \subset \mathbf{R}^{n}$. We recall that a function $u$ on an open set in $\mathbf{R}^{n}$ is quasi-convex if the function $u(x)+c|x|^{2}$ is convex for some $c>0$. Local quasi-convexity is invariant under coordinate changes and therefore makes sense on manifolds.

Suppose $u$ is locally quasi-convex on $X$. Then

$$
u \in \mathbf{F}(X) \quad \Longleftrightarrow \quad D_{x}^{2} u \in \mathbf{F} \text { a.e. on } X .
$$

If $\mathbf{F}+\mathbf{G} \subset \mathbf{H}$, then for quasi-convex functions $u$ and $v$,

$$
u \in \mathbf{F}(X) \text { and } v \in \mathbf{G}(X) \quad \Rightarrow \quad u+v \in \mathbf{H}(X) .
$$

Both of these results hold in much greater generality.

TheOrem 5.3.1. (AE Theorem) Suppose F is a subequation (in the sense of Definition 2.2.1) on a manifold $X$, and suppose $u$ is locally quasi-convex on $X$. Then

$$
u \in F(X) \quad \Longleftrightarrow \quad J_{x}^{2} u \in F_{x} \text { a.e. on } X .
$$

Theorem 5.3.2. (Quasi-Convex Addition) Given three subequations F, $G$ and $H$ (as in 5.3.1) with $F+G \subset H$, one has that

$$
u \in F(X) \text { and } v \in G(X) \quad \Rightarrow \quad u+v \in H(X) .
$$

for locally quasi-convex functions $u$ and $v$.

Theorem 5.3.1 follows in an elementary manner from either Jensen's Lemma $\left[\mathrm{J}_{1}\right]$ or Slodkowski's Lemma $\left[\mathrm{S}_{1}\right]$ (in fact, they are equivalent). Theorem 5.3.2 is immediate from the first. These results will be elaborated in a forthcoming paper.

Of course, quasi-convex approximation can be used in the constant coefficient case to obtain the full Addition Theorem:

$$
u \in \mathbf{F}(X) \text { and } v \in \mathbf{G}(X) \quad \Rightarrow \quad u+v \in \mathbf{H}(X) .
$$

Application 5.3.3. (Comparison via Monotonicity for Constant Coefficient Equations) Suppose $\mathbf{F}$ satisfies

$$
\mathbf{F}+\mathbf{M} \subset \mathbf{F}
$$

where $\widetilde{\mathbf{M}}$-subharmonic functions satisfy the Zero Maximum Prinicple. From (5.3.2) we have $\mathbf{F}+\widetilde{\mathbf{F}} \subset \widetilde{\mathbf{M}}$. Therefore

$$
u \in \mathbf{F}(X) \quad \text { and } \quad v \in \widetilde{\mathbf{F}}(X) \quad \Rightarrow \quad u+v \in \widetilde{\mathbf{M}}(X),
$$

and so comparison holds for $\mathbf{F}$. 
Note that $\mathbf{M}$ can be any of the monotonicity cones discussed in Appendix B. For example, the cone $\mathbf{M}=\mathbf{R}_{-} \times \mathbf{R}^{n} \times \mathcal{P}$ implies comparison for all gradient independent subequations.

\section{Removable Singularities}

Monotonicity cones lend themselves nicely to the question of removable singularities for $F$-subharmonic and $F$-harmonic functions.

6.1. $M$-Polar Sets. Suppose $M \subset J^{2}(X)$ is a convex cone subequation, i.e., one for which the fibres are convex cones with vertex at the origin.

Definition 6.1.1. A closed subset $E \subset X$ is called $C^{\infty} M$-polar if $E=\{x: \psi(x)=-\infty\}$ for some $M$-subharmonic function $\psi$ which is smooth on $X-E$.

\section{Examples.}

(a) Consider the pure second-order constant coefficient equation $\mathbf{M}=$ $\mathcal{P}$ on $\mathbf{R}^{n}$. The $\mathcal{P}$-subharmonic functions are convex (See Proposition 2.1.7), and so there do not exist any $C^{\infty} \mathcal{P}$-polar sets.

(b) Consider the complex analogue $\mathcal{P}^{\mathbf{C}}$ on $\mathbf{C}^{n}$. Then $\mathcal{P}^{\mathbf{C}}$-subharmonic functions are the standard plurisubharmonic functions and $\mathcal{P}^{\mathbf{C}}$ polar sets are standard pluripolar sets. These exist is abundance. They include, for example, $\log |f|$ with $f$ holomorphic.

(c) For the quaternionic analogue $\mathcal{P}^{\mathbf{H}}$ on $\mathbf{H}^{n}$ there is a 2-sphere of complex structures coming from unit imaginary quaternions. A plurisubharmonic function in any one of these structures is $\mathcal{P}^{\mathbf{H}}$ subharmonic, and so any pluripolar set for that structure is $\mathcal{P}^{\mathbf{H}}$ polar.

(d) Consider the constant coefficient subequation $\mathcal{P}(p)$ defined in (4.3.1) and equal to $\mathbf{F}\left(G\left(p, \mathbf{R}^{n}\right)\right)$ for integer $p$ (cf. 2.1.11(c)). The following result is proved in $\left[\mathrm{HL}_{12}\right]$ using the theory of classical Riesz potentials (see [L] for example).

TheOREM 6.1.2. Any closed set of locally finite Hausdorff $(p-2)$-measure is $\mathcal{P}(p)$-polar.

6.2. Removability Results. The following removable singularity results on manifolds are proved in $\left[\mathrm{HL}_{12}\right]$. Recall that $M$ is a monotonicity cone for $F$ if and only if it is a monotonicity cone for $\widetilde{F}$ (see 4.2.2).

Theorem 6.2.1. Suppose $F$ is a subequation on $X$ with monotonicity cone $M$, and $E \subset X$ is locally $C^{\infty} M$-polar with no interior. Then $E$ is removable for $F$-subharmonic functions which are locally bounded above across $E$. More precisely, if $u \in F(X-E)$ is locally bounded across $E$, then its canonical upper semi-continuous extension $U$ to $X$ is $F$-subharmonic on $X$. 
TheOrem 6.2.2. Suppose $F$ is a subequation on $X$ with monotonicity cone $M$, and $E \subset X$ is locally $C^{\infty} M$-polar with no interior. Then for $u \in C(X)$

$u$ is F-harmonic on $X-E \quad \Rightarrow \quad u$ is F-harmonic on $X$.

More generally, Theorem 6.2.1 remains true when $E$ has interior if the extension $U$ is defined to be $\equiv-\infty$ on $\operatorname{Int} E$.

Theorems 6.2.1 and 6.2.2 can be applied to the many subequations given in Section 4.3. For example, this gives removable singularity results for all branches of the homogeneous complex Monge-Ampère equation on a complex hermitian manifold. Here $E$ can be any pluripolar set (not just a $C^{\infty}$ pluripolar set). The result also applies to the intrinsic notion of maximal functions on an almost complex manifold (see $\left[\mathrm{HL}_{10}\right]$ ).

These general results combined with Theorem 6.1 .2 above give the following. We restrict attention to constant coefficient pure second-order subequations in $\mathbf{R}^{n}$.

Corollary 6.2.3. If $F$ is a subequation for which $\mathcal{P}(p)$ is a monotonicity cone, then any closed set of locally finite Hausdorff $(p-2)$-measure is removable for $F$ - and $\widetilde{F}$-subharmonics and $F$-harmonics as in the two theorems above.

This applies immedately to all branches of the equation $\mathrm{MA}_{p}$ in Example 4.3.2. It also applies to all subequations geometrically defined by a subset $\mathbb{G}$ of the Grassmannian $G\left(p, \mathbf{R}^{n}\right)$. (See Example 2.1.11 and also example (c) following Theorem 2.1.12.). These include the Lagrangian and Special Lagrangian subequations in $\mathbf{C}^{n}$, the associative and coassociative subequations in $\mathbf{R}^{7}$, and the Cayley subequations in $\mathbf{R}^{8}$ (where the appropriate value of $p$ is clear in each case).

For the general applicability of this result we introduce the following invariant, which is studied in $\left[\mathrm{HL}_{15}\right]$.

Definition 6.2.4. Suppose $M$ is a convex cone subequation. The Riesz characteristic $p_{M}$ of $M$ is defined to be

$$
p_{M} \equiv \sup \left\{p \in \mathbf{R}: I-p P_{e} \in M \forall|e|=1\right\} .
$$

It has the important property that

$$
\mathcal{P}(p) \subset M \quad \Longleftrightarrow \quad p \leq p_{M} .
$$

and hence: For any subequation $F$ which is $M$-monotone, closed sets of locally finite Hausdorff $\left(p_{M}-2\right)$-measure are $F$-removable as above.

Example 6.2.5. For $M=\mathbf{P}_{\lambda, \Lambda}$, the Pucci cone defined in (4.5.2), the Riesz characteristic is

$$
p_{M}=\frac{\lambda}{\Lambda}(n-1)+1
$$


As a consequence one retrieves the removable singularity results in $[\mathrm{AGV}]$. In fact Corollary 6.2.3 is stronger since it applies to interesting equations which are not uniformly elliptic.

For $M=\mathcal{P}(\delta)$, another choice for defining uniform ellipticity, the Riesz characteristic is

$$
p_{M}=\frac{\delta n+1}{\delta+1}
$$

Final Remark. In the special case of convex subequations (in the general setting of manifolds) there are many interesting removability results $\left[\mathrm{HL}_{12}\right]$. They come from combining the Strong Bellman Principle (see §10) and known results ([Le], $\left[\mathrm{HP}_{1,2}\right],[\mathrm{H}]$, [Shi]) for linear elliptic equations. See $\left[\mathrm{HL}_{13}\right]$ for details.

\section{Boundary Convexity}

Fix a subequation $F$ on a manifold $X$ and a domain $\Omega \subset \subset X$ with smooth boundary. We shall be interested in the Dirichlet problem for $F$ harmonic functions on $\Omega$. In this chapter we present geometric conditions on $\partial \Omega$ which guarantee the existence of solutions for all continuous boundary functions. These conditions are based on the following concept.

7.1. The Asymptotic Interior of a Reduced Subequation. Throughout this section we assume that $F$ is a subequation which is "independent of the $r$-variable" or "reduced". This means that with respect to the splitting

$$
J^{2}(X)=\mathbf{R} \oplus J_{\text {red }}^{2}(X)
$$

in (3.1.1), $F$ is of the form $F=\mathbf{R} \times F_{0}$. For simplicity we just take $F \subset J_{\text {red }}^{2}(X)$.

Definition 7.1.1. The asymptotic interior $\vec{F}$ of $F$ is the set of all $J \in J_{\text {red }}^{2}(X)$ for which there exists a neighborhood $\mathcal{N}(J)$ in the total space of $J_{\text {red }}^{2}(X)$ and a number $t_{0}>0$ such that

$$
t \cdot \mathcal{N}(J) \subset F \text { for all } t \geq t_{0}
$$

The set $\vec{F}$ is an open cone in $J_{\text {red }}^{2}(X)$ which satisfies Condition $(\mathrm{P})$. If $F$ is itself a cone, then $\vec{F}=\operatorname{Int} F$. Otherwise, $\vec{F}$ is smaller than $\operatorname{Int} F$ and may be empty.

Definition 7.1.2. A function $u \in C^{2}(X)$ is called strictly $\vec{F}$ subharmonic if $J_{\text {red, } x}^{2} u \in \vec{F}$ for all $x$.

Let $\Omega \subset X$ be a domain with smooth boundary $\partial \Omega$. By a defining function for $\partial \Omega$ we mean a smooth function $\rho$ defined on a neighborhood of $\partial \Omega$ such that $\partial \Omega=\{x: \rho(x)=0\}, d \rho \neq 0$ on $\partial \Omega$, and $\rho<0$ on $\Omega$. 
Definition 7.1.3. Suppose $F$ is a reduced subequation. The boundary $\partial \Omega$ is said to be strictly $F$-convex at $x \in \partial \Omega$ if there exists a strictly $\vec{F}$-subharmonic defining function for $\partial \Omega$ on some neighborhood of $x$

This is equivalent to either of the following two conditions.

(i) For some local defining function $\rho, J_{\text {red } x}^{2} \rho \in \vec{F}$.

(ii) For any local defining function $\rho, \quad J_{\text {red }, x}^{2} \rho+t(d \rho)_{x} \circ(d \rho)_{x} \in \vec{F}$ for all $t \geq$ some $t_{0}$.

7.2. General $F$-Convexity. Suppose now that $F \subset J^{2}(X)$ is a general subequation on $X$. For each $\lambda \in \mathbf{R}$ there is a reduced subequation $F_{\lambda} \subset J_{\text {red }}^{2}(X)$ obtained by fixing the $r$-variable to be $\lambda$, that is

$$
F_{\lambda} \equiv F \cap\left(\{\lambda\} \times J_{\text {red }}^{2}(X)\right) .
$$

As above we fix a domain $\Omega \subset X$ with smooth boundary $\partial \Omega$.

Definition 7.2.1. Suppose $F$ is a general subequation. The boundary $\partial \Omega$ is said to be strictly $F$-convex at $x \in \partial \Omega$ if it is strictly $\overrightarrow{F_{\lambda}}$-convex at $x$ for all $\lambda \in \mathbf{R}$.

For example, consider the universal riemannian subequation $F$ given by Hess $u \geq 0$ and $\operatorname{det}\{\operatorname{Hess} u\} \geq e^{u}$. Then $F_{\lambda}$ is given by the condition that Hess $u \geq 0$ and $\operatorname{det}\{\operatorname{Hess} u\} \geq e^{\lambda}$. One easily checks that for every $\lambda, \overrightarrow{F_{\lambda}}$ is the open cone $\{$ Hess $u>0\}$, and so in this case the strictly $F$-convex boundaries are just the classical strictly convex boundaries.

Strict $F$ - and $\widetilde{F}$-convexity of $\partial \Omega$ at each point are sufficient for the construction of barriers used in the proof of the existence of solutions to the Dirichlet problem.

7.3. F-Convexity in Terms of the Second Fundamental Form. For a reduced subequation $F$ on a riemannian manifold $X$, the $F$-convexity of a boundary $\partial \Omega$ can be characterized in terms of its second fundamental form $I I_{\partial \Omega}$ with respect to the outward-pointing unit normal $\nu$. We use the decomposition given by $(2.2 .4)$ :

$$
J_{\text {red }}^{2}(X)=T^{*} X \oplus \operatorname{Sym}^{2}\left(T^{*} X\right) .
$$

Proposition 7.3.1. The boundary $\partial \Omega$ is strictly F-convex at $x \in \partial \Omega$ if and only if

$$
\left(\nu, t P_{\nu} \oplus I I_{\partial \Omega}\right) \in \overrightarrow{F_{x}} \quad \text { for all } t \geq \text { some } t_{0} .
$$

where $P_{\nu}$ denotes orthogonal projection onto the normal line $\mathbf{R} \nu$ at $x$.

Note. Blocking with respect to the decomposition $T_{x} X=\mathbf{R} \nu \oplus T_{x}(\partial \Omega)$, (7.3.1) can be rewritten

$$
\left((1,0),\left(\begin{array}{cc}
t & 0 \\
0 & I I_{\partial \Omega}
\end{array}\right)\right) \in \overrightarrow{F_{x}} \quad \text { for all } t \geq \text { some } t_{0} .
$$


7.4. Examples. (a) $k$-Laplacians. There are many examples where every boundary is strictly $F$-convex. The simplest one is the subequation $\Delta u \geq 0$ or more generally $\Delta u \geq f(x, u)$ where $f$ is non-decreasing in $u$.

Other examples come from the constant coefficient $k$-Laplace subequation, defined by

$$
\mathbf{F}_{k}^{\text {Lap }} \equiv \text { Closure }\left\{(p, A):|p|^{2} \operatorname{tr} A+(k-2) p^{t} A p>0\right\}
$$

where $k \geq 1$. These equations are self-dual. Since $\mathbf{F}_{k}^{\text {Lap }}$ is a cone, $\overrightarrow{\mathbf{F}}_{k}^{\text {Lap }}=$ Int $\mathbf{F}_{k}^{\text {Lap }}$. One can check directly from (7.3.2) that for $k>1$ every boundary is $\overrightarrow{\mathbf{F}}_{k}^{\text {Lap }}$-convex.

When $k=1$ this equation is the implicit minimal surface equation studied by De Giorgi and his school [Giu]. Here one sees that a boundary $\partial \Omega$ is strictly $\mathbf{F}_{1}^{\text {Lap }}$-convex if and only if it is strictly mean convex, i.e., $\operatorname{tr}\left(I I_{\partial \Omega}\right)>0$ at all points.

At the other extreme is the infinity Laplacian (cf. [CIL], [ $\left.\mathrm{J}_{2}\right],[\mathrm{ESm}]$ )

$$
\mathbf{F}_{\infty}^{\text {Lap }} \equiv \text { Closure }\left\{(p, A): p^{t} A p>0\right\}
$$

where again all boundaries are strictly $\mathbf{F}_{\infty}^{\mathrm{Lap}}$-convex.

(b) Elementary Symmetric Functions of $\operatorname{Hess}(u)$. Consider Example 2.1.2(c)

$$
\mathbf{F}_{\sigma_{k}} \equiv\left\{\sigma_{k}(A) \geq 0, \sigma_{k-1}(A) \geq 0, \ldots, \sigma_{1}(A) \geq 0\right\}
$$

which can be extended to the complex and quaternionic cases, and carried over to riemannian manifolds. One finds that $\partial \Omega$ is strictly $\mathbf{F}_{\sigma_{k}}$-convex if and only if

$$
\sigma_{k-1}\left(I I_{\partial \Omega}\right)>0, \quad \sigma_{k-2}\left(I I_{\partial \Omega}\right)>0, \ldots, \sigma_{1}\left(I I_{\partial \Omega}\right)>0 .
$$

Moreover, if $\partial \Omega$ is strictly $\mathbf{F}_{\sigma_{k}}$-convex, then it is $\mathbf{F}_{\sigma_{k}, i}$-convex for every branch $\mathbf{F}_{\sigma_{k}, i}$ of the equation $\sigma_{k}$ (Hess $\left.u\right)=0$ (see Section 4.3). This includes the dual subequation $\widetilde{\mathbf{F}}_{\sigma_{k}}$, which is the bottom branch.

(c) Geometrically Defined Subequations. Consider now the subequations discussed in Example 2.1.11. Here the boundary convexity is particularly nice. Fix a compact subset $\mathbb{G} \subset G\left(p, \mathbf{R}^{n}\right)$ and define $\mathbf{F}(\mathbb{G})$ as in (2.1.6). Then a boundary $\partial \Omega$ is strictly $\mathbf{F}(\mathbb{G})$-convex if and only if

$\operatorname{tr}_{W}\left\{I I_{\partial \Omega}\right\}>0$ for all $\mathbb{G}$ planes $W$ which are tangent to $\partial \Omega$.

This condition holds automatically at $x \in \partial \Omega$ if there are no $\mathbb{G}$-planes tangent to $\partial \Omega$ at $x$.

On the other hand, if $\mathbb{G}=G\left(p, \mathbf{R}^{n}\right)$, then $\partial \Omega$ is strictly $F(\mathbb{G})$-convex if and only if $I I_{\partial \Omega}$ has positive trace on all tangent $p$-planes, i.e., $\partial \Omega$ is $p$-convex as in $[\mathrm{Wu}],\left[\mathrm{Sha}_{1,2}\right]$.

For example, suppose $\mathbb{G} \subset G\left(1, \mathbf{R}^{2}\right)$ is the single point $\mathbb{G}=\{x$-axis $\}$. Then a domain $\Omega \subset \subset \mathbf{R}^{2}$ with smooth boundary is strictly $\mathbb{G}$-convex iff the curvature vector of $\partial \Omega$ points strictly inward at every horizontal tangent. 
This implies that all horizontal slices of $\Omega$ are connected. Thus, one can see directly that the Dirichlet problem for $\mathbb{G}$-harmonic functions $\left(u_{x x}=0\right)$ is uniquely solvable for all continuous boundary data.

A classical example comes from the set $\mathbb{G}=G_{\mathbf{C}}\left(1, \mathbf{C}^{n}\right) \subset G\left(2, \mathbf{R}^{2 n}\right)$ of complex lines in $\mathbf{C}^{n}$. A domain $\Omega \subset \mathbf{C}^{n}$ is strictly $\mathbb{G}$-convex iff it is strictly pseudo-convex in the usual sense in complex analysis (cf. $\left.\left[\mathrm{Ho}_{1}\right]\right)$. This is the boundary convexity required to solve the Dirichlet problem for $\mathcal{P}^{\mathbf{C}}=\mathbf{F}(\mathbf{G})$ harmonic functions, i.e., for solutions to the homogeneous complex MongeAmpère equation.

We note that in all cases $F(\mathbb{G}) \subset \widetilde{F(\mathbb{G})}$, so that a strictly $F(\mathbb{G})$-convex boundary is automatically strictly $\widetilde{F(\mathbb{G})}$-convex.

(d) p-Plurisubharmonic Functions. Consider now the $p^{\text {th }}$ branch of the homogeneous complex Monge-Ampère equation. This is the pure second-order subequation given by $\Lambda_{p}^{\mathbf{C}} \equiv\left\{A: \lambda_{p}^{\mathbf{C}}(A) \geq 0\right\}$ where $\lambda_{1}^{\mathbf{C}}(A) \leq$ $\cdots \leq \lambda_{n}^{\mathbf{C}}(A)$ are the ordered eigenvalues of the hermitian symmetric part of $A$ (see 2.1.3 and 2.1.10). The $\Lambda_{p}^{\mathrm{C}}$-subharmonic functions are the classical $(p-1)$-plurisubharmonic functions in complex analysis - those for which the complex hessian has at least $n-p+1$ non-negative eigenvalues. The Dirichlet problem for $\Lambda_{p}^{\mathbf{C}}$-harmonic functions was studied by Hunt and Murray [HM] and then solved by Slodkowski $\left[\mathrm{S}_{1}\right]$. A smooth boundary $\partial \Omega \subset \mathbf{C}^{n}$ is strictly $\Lambda_{p}^{\mathrm{C}}$-convex iff

$$
\lambda_{p}^{\mathbf{C}}\left(I I_{\partial \Omega}\right) \geq 0, \quad \text { or equivalently }
$$

the Levi form of $\partial \Omega$ has $n-p-1$ eigenvalues $\geq 0$ at each point.

(e) Calabi-Yau-Type Equations. Let $X$ be a complex hermitian manifold. Consider the subequation $F$ on $X$ corresponding to $\operatorname{det}_{\mathbf{C}}(I+$ $\left.\operatorname{Hess}_{\mathbf{C}} u\right) \geq f(x, u)$ for a continuous $f>0$ which is non-decreasing in $u$ and $I+$ Hess $u \geq 0$. For $\lambda \in \mathbf{R}$ the subequation $F_{\lambda}$ given in Section 7.2 corresponds to $\operatorname{det}_{\mathbf{C}}\left(I+\operatorname{Hess}_{\mathbf{C}} u\right) \geq f(x, \lambda)$ at each point. One checks that $F_{\lambda}$-convexity of a boundary $\partial \Omega$ amounts to the statement that $\left(I I_{\partial \Omega}\right)_{\mathbf{C}}>-I$ at each point (a condition independent of $\lambda$ ). Levi convexity of the boundary $\left(\left(I I_{\partial \Omega}\right)_{\mathbf{C}}>0\right)$ will certainly suffice.

(f) Principal curvatures of the graph. Other equations of interest are those which impose conditions on the principal curvatures of the graph of the function $u$ in $X \times \mathbf{R}$. See $\left[\mathrm{HL}_{6}, \S 11.5\right]$ for a complete discussion of this case.

\section{The Dirichlet Problem}

Throughout this chapter $F \subset J^{2}(X)$ will be a subequation on a manifold $X$ and $\Omega \subset \subset X$ will be a domain with smooth boundary $\partial \Omega$. We shall say that existence holds for the Dirichlet Problem for $F$-harmonic functions on $\Omega$ if for each continuous function $\varphi \in C(\partial \Omega)$ there exists a function $u \in C(\bar{\Omega})$ such that 
(i) $u$ is $F$-harmonic on $\Omega$, and

(ii) $\left.u\right|_{\partial \Omega}=\varphi$.

We say that uniqueness holds for this problem if for each $\varphi \in C(\partial \Omega)$, there exists at most one such function $u$.

8.1. General Theorems. It is an elementary fact that if comparison holds for $F$ on $X$ (see Definition 5.1), then uniqueness holds for the Dirichlet problem. Under appropriate boundary convexity comparison also implies existence.

TheOrem 8.1.1. Suppose comparison holds for $F$ on $X$. Then existence and uniqueness hold for the Dirichlet problem for $F$-harmonic functions on any domain $\Omega \subset \subset X$ whose boundary is both strictly F-convex and strictly $\widetilde{F}$-convex.

Note that $u$ is $F$-harmonic if and only if $-u$ is $\widetilde{F}$-harmonic. Thus, it is expected that both conditions, strict $F$ and $\widetilde{F}$ convexity, should be required, if one of them is. Often one of these convexity conditions implies the other. This is clearly the case for $F=\mathcal{P}$ in $\mathbf{R}^{n}$ where strict $\mathcal{P}$-convexity is the usual strict convexity and $\widetilde{\mathcal{P}}$-convexity is much weaker. It also holds in the case of $q$-plurisubharmonic functions (Example $7.4(\mathrm{~d})$ ) where by $(7.3 .7) \mathcal{P}_{q}^{\mathrm{C}}$ convexity implies $\mathcal{P}_{q^{\prime}}^{\mathbf{C}}$-convexity if $q<q^{\prime}$. This is reflected in the work of Hunt and Murray [HM] who noted the failure of the statement when only one convexity condition is required.

Theorems 5.1.2 and 5.2.1 imply that

If local weak comparison and strict approximation hold for $F$ on $X$, then comparison holds for $F$ on $X$.

TheOREm 8.1.2. Let $F$ be a subequation with monotonicity cone $M$. Suppose that:

(i) F is locally affinely jet-equivalent to a constant coefficient subequation, and

(ii) $X$ carries a strictly $M$-subharmonic function.

Then existence and uniqueness hold for the Dirichlet problem for $F$ harmonic functions on any domain $\Omega \subset \subset X$ whose boundary is both strictly $F$ - and $\widetilde{F}$-convex.

Comparison and therefore uniqueness follow from Theorems 5.1.3 and 5.2.2. It is then proved, using comparison and barriers constructed from boundary convexity, that existence also holds. Further details are given in $\S 8$.

Assumption (ii) is always true for pure second-order equations in $\mathbf{R}^{n}$ (and in any complete simply-connected manifold of non-positive sectional curvature) since the subequation $\mathcal{P}$ is always a monotonicity cone by the positivity condition $(\mathrm{P})$ and $|x|^{2}$ is strictly $\mathcal{P}$-convex. 
On the other hand something like assumption (ii) must be required in the general case. For example, suppose $F$ is a universal riemannian equation as in 2.2.3. One could completely change the geometry (and topology) of the interior of a domain $\Omega \subset X$ without changing the $F$-convexity of the boundary. Take the subequation $\mathcal{P}$ on the euclidean ball, and change the interior so that it is not contractible. Then there are no $\mathcal{P}$-subharmonic (riemannian convex) functions on the resulting space, and certainly no $\mathcal{P}$ harmonic ones.

In homogeneous spaces one can apply a trick of Walsh [W] to establish existence without uniqueness.

Theorem 8.1.3. Let $X=G / H$ be a riemannian homogeneous space and suppose that $F \subset J^{2}(X)$ is a subequation which is invariant under the natural action of $G$ on $J^{2}(X)$. Let $\Omega \subset \subset X$ be a connected domain whose boundary is both $F$ and $\widetilde{F}$ strictly convex. Then existence holds for the Dirichlet problem for F-harmonic functions on $\Omega$.

This theorem applies to give (the known) existence for the $k$-Laplacian, $1<k \leq \infty$ on arbitrary domains, and for the 1-Laplacian on mean convex domains in $G / H$. The literature on these equations in $\mathbf{R}^{n}$ is vast. See [JLM], [CIL], $\left[\mathrm{J}_{2}\right],[\mathrm{ESm}]$ and references therein, for example. We note that even in $\mathbf{R}^{n}$, uniqueness for the 1-Laplacian fails catastrophically. For a generic smooth function on the boundary of the unit disk in $\mathbf{R}^{2}$ there are families of solutions to the Dirichlet problem parameterized by $\mathbf{R}$ (and often $\mathbf{R}^{m}$ for large $m)$ !

The proof of existence in the theorems above uses the standard Perron method based on the properties in Theorem 2.3.1. Given $\varphi \in C(\partial \Omega)$, consider the family

$$
\mathcal{F}(\varphi) \equiv\{u \in \operatorname{USC}(\bar{\Omega}) \cap F(\Omega): u \leq \varphi \text { on } \partial \Omega\},
$$

and define the Perron function to be the upper envelope of this family:

$$
U(x) \equiv \sup _{u \in \mathcal{F}(\varphi)} u(x) .
$$

Proposition 8.1.4. Suppose that $F$ satisfies weak comparison and that $\partial \Omega$ is both $F$ and $\widetilde{F}$ strictly convex. Then the upper and lower semicontinuous regularizations $U^{*}$ and $U_{*}$ of $U$ on $\bar{\Omega}$ satisfy:

(i) $U^{*}=U_{*}=U=\varphi$ on $\partial \Omega$,

(ii) $U=U^{*}$ on $\bar{\Omega}$

(iii) $U$ is $F$-subharmonic and $-U_{*}$ is $\widetilde{F}$-subharmonic on $\Omega$.

The classical barrier argument, used by Bremermann [B] for the case $F=\mathcal{P}^{\mathbf{C}}$, establishes (i), while weak comparison is used in (ii). Part (iii) relies on a "bump argument" found in Bedford and Taylor $\left[\mathrm{BT}_{1}\right]$ and also in $[\mathrm{I}]$.

When one can ultimately establish comparison, as in Theorem 8.1.2, the Perron function is the unique solution. When this is not necessarily possible, 
as in Theorem 8.1.3, arguments of Walsh [W] can be applied to show that the Perron function is a solution.

In this latter case one can say more. Fix $F$ and $\Omega$ as in Theorem 8.1.3.

Suppose

$U$ is the Perron function for $F$ on $\Omega$ with boundary values $\varphi$, and

$-\widetilde{U}$ is the Perron function for $\widetilde{F}$ on $\Omega$ with boundary values $-\varphi$.

Both $U$ and $\widetilde{U}$ solve the Dirichlet problem for F-harmonic functions on $\Omega$ with boundary values $\varphi$, and if $u$ is any other such solution,

$$
\widetilde{U} \leq u \leq U
$$

Theorems 8.1.2 and 8.1.3 have wide applications. In the following sections we will examine some specific examples.

8.2. Manifolds with Reduced Structure Group. Fix a constant coefficient subequation $\mathbf{F} \subset \mathbf{J}^{2}$, and let

$$
G \equiv G_{\mathbf{F}} \equiv\{g \in \mathrm{O}(n): g(\mathbf{F})=\mathbf{F}\}
$$

where $g$ acts naturally on $\mathbf{J}^{2}$ by $g(r, p, A)=\left(r, g p, g^{t} A g\right)$.

Definition 8.2.1. Let $X$ be a riemannian $n$-manifold and $G \subset \mathrm{O}(n)$ a subgroup. A topological $G$-structure on $X$ is a family $\left\{\left(U_{\alpha}, e_{\alpha}\right)\right\}_{\alpha}$ where $\left\{U_{\alpha}\right\}_{\alpha}$ is an open covering of $X$ and each $e_{\alpha}=\left(e_{\alpha}^{1}, \ldots, e_{\alpha}^{n}\right)$ is a continuous tangent frame field on $U_{\alpha}$, such that for all $\alpha, \beta$ the change of framing $g: U_{\alpha} \cap U_{\beta} \rightarrow \mathrm{O}(n)$ takes values in $G$.

Each constant coefficient subequation $\mathbf{F}$ canonically determines a subequation $F$ on any riemannian manifold $X$ equipped with a topological $G_{\mathbf{F}^{-}}$ structure. (Use the splitting (2.2.4) and then the trivializations induced by the local tangent frames. The subequation determined by $\mathbf{F}$ in these trivializations is preserved under the change of framings.) By Proposition 3.2.5, $F$ is locally jet-equivalent to $\mathbf{F}$.

If $\mathbf{M}$ is a $G_{\mathbf{F}}$-invariant monotonicity cone for $\mathbf{F}$, then the corresponding subequation $M$ on $X$ is a monotonicity cone for $F$. Note that the maximal monotonicity cone for $\mathbf{F}$ is always $G_{\mathbf{F}}$-invariant.

THEOREM 8.2.2. Let $F$ be a subequation with monotonicity cone $M$ canonically determined by $\mathbf{F}$ and $\mathbf{M}$ on a riemannian manifold $X$ with a topological $G_{\mathbf{F}}$-structure. Let $\Omega \subset \subset X$ be a domain with smooth boundary which is both $F$ and $\widetilde{F}$ srictly convex. Assume there exists a strictly $M$ subharmonic function on $\bar{\Omega}$. Then the Dirichlet Problem for F-harmonic functions is uniquely solvable for all $\varphi \in C(\partial \Omega)$.

EXAmple 8.2.3. (a) Universal Riemannian Subequations: As noted in Remark 2.2.3, if $G_{\mathbf{F}}=\mathrm{O}(n)$, then $\mathbf{F}$ universally determines a subequation on every riemannian manifold by choosing the framings $e_{\alpha}$ to be orthonormal. In particular this covers all branches of the homogeneous 
Monge-Ampère equation. In fact, it covers all pure second-order subequations which depend only on the ordered eigenvalues of the Hessian. The subequation $\mathcal{P}=\{$ Hess $u \geq 0\}$ is a monotonicity cone for all such equations. Thus Theorem 8.2.2 applies to all such $F$ 's in any region of $X$ where there exists a smooth strictly convex function.

Other interesting examples are given by the branches of the $p$-convex Monge-Ampère equation $\mathrm{MA}_{p}$ given in example 4.3.2. Here the monotonicity cone is $\mathcal{P}(p)$, and the appropriate boundary convexity is the $p$-convexity discussed in 7.4 (c).

Further examples come from elementary symmetric functions of Hess $u$ (see 7.4 (b) and the discussion after 4.3.5.), and functions of eigenvalues of the graph $(7.4$ (f)).

(b) Universal Hermitian Subequations: If $G_{\mathbf{F}}=\mathrm{U}(n)$, then $\mathbf{F}$ universally determines a subequation on every almost complex hermitian manifold. For example, this covers all pure second-order subequations which depend only on the ordered eigenvalues of the hermitian symmetric part Hess $\mathbf{C} u$ of Hess $u$. For such equations, $\mathcal{P}^{\mathbf{C}}=\left\{\operatorname{Hess}_{\mathbf{C}} u \geq 0\right\}$ is a monotonicity cone. Thus, for example, one has the following consequence of Theorem 8.2.2. Let $X$ be an almost complex hermitian manifold, and $\Omega \subset \subset X$ a smoothly bounded domain with a strictly plurisubharmonic $\left(\mathcal{P}^{\mathrm{C}}\right.$-subharmonic) defining function. Then the Dirichlet problem for every branch of the homogeneous complex Monge-Ampère equation is uniquely solvable on $\Omega$.

A similar result holds for branches of the equation $\mathrm{MA}_{p}^{\mathbf{C}}$ where $p$ convexity of the Levi form on the boundary plays a role (see $7.4(\mathrm{~d})$ ).

The discussion of elementary symmetric functions also carries over to this case.

Theorem 8.2.2 can similarly be applied to Calabi-Yau type equations (7.4 (e)).

All of this discussion can be replicated for almost quaternionic hermitian manifolds.

(c) Geometrically Defined Subequations: Theorem 8.2.2 applies directly to all subequations geometrically defined by a compact subset $\mathbb{G} \subset$ $G\left(p, \mathbf{R}^{n}\right)$ (see 2.1.11, 2.1.12 and 7.4 (b)). Suppose $X$ has a topological $G$ structure where $G=\{g \in \mathrm{O}(n): g(\mathbb{G})=\mathbb{G}\}$ and let $F(\mathbb{G})$ be the corresponding subequation on $X$. Suppose $\Omega \subset X$ is a domain with a global defining function which is strictly $\mathbb{G}$-plurisubharmonic. Then the Dirichlet problem for $\mathbb{G}$-harmonic functions is uniquely solvable on $\Omega$.

Thus, one can solve the Dirichlet problem for (in fact, all branches of) the Lagrangian harmonic equation (see 2.1.11 (d)) on domains with a strictly Lagrangian-plurisubharmonic defining function.

One can also solve for $G(\varphi)$-harmonic functions on strictly $G(\varphi)$-convex domains in a manifold with a topological calibration $\varphi$. A typical example is the following. Let $X$ be a riemannian 7 -manifold with a topological $\mathrm{G}_{2^{-}}$ structure determined by a global associative 3 -form $\varphi$ of constant comass 1 . 
(Such structures exist on $X$ if and only if $X$ is a spin manifold.) Then the Dirichlet problem for $G(\varphi)$-harmonic functions is uniquely solvable on any domain with a strictly $G(\varphi)$-plurisubharmonic defining function.

8.3. Inhomogeneous Equations. Since Theorem 8.1.2 assumes affine jet-equivalence, it applies to inhomogeneous equations as in Examples 3.2.78 . In these cases boundary convexity and monotonicity cones are the same as in the homogeneous case.

8.4. Existence Without Uniqueness. Theorem 8.1.3 applies in cases where monotonicity cones do not exist, such as the 1-laplacians in 7.4 (a). As previously noted, solutions of the Dirichlet problem for the 1laplacian are highly non-unique. However, they are all caught between the Perron functions $U$ and $\widetilde{U}$ (see (8.1.2) above).

8.5. Parabolic Equations. The methods and results above carry over effectively to parabolic equations. Let $X$ be a riemannian $n$-manifold with a topological $G$-structure for $G \subset \mathrm{O}(n)$, and consider a constant coefficient subequation of the form

$$
\mathbf{F}=\left\{J \in \mathbf{J}^{2}: f(J) \geq 0\right\}
$$

where $f: J^{2}(X) \rightarrow \mathbf{R}$ is $G$-invariant, $\mathcal{P}$ - and $\mathcal{N}$-monotone, and Lipschitz in the reduced variables $(p, A)$. This induces a subequation $F$ on $X$. The associated constant coefficient parabolic subequation $\mathbf{H}_{\mathbf{F}}$ on $\mathbf{R} \times \mathbf{R}^{n}$ is defined by

$$
f(J)-p_{0} \geq 0
$$

(where $p_{0}$ denotes the $u_{t}$ component of the 2 -jet of $u$ ), and it induces the associated parabolic subequation $H_{F}$ on the riemannian product $\mathbf{R} \times X$. The $H_{F}$-harmonic functions are solutions of the equation

$$
u_{t}=f\left(u, D u, D^{2} u\right) \text {. }
$$

Examples which can be treated include:

(i) $f=\operatorname{tr} A$, the standard heat equation $u_{t}=\Delta u$ for the LaplaceBeltrami operator.

(ii) $f=\lambda_{q}(A)$, the $q$ th ordered eigenvalue of $A$. This is the natural parabolic equation associated to the $q$ th branch of the MongeAmpère equation.

(iii) $f=\operatorname{tr} A+\frac{k}{|p|^{2}+\epsilon^{2}} p^{t} A p$ for $k \geq-1$ and $\epsilon>0$. When $X=\mathbf{R}^{n}$ and $k=-1$, the solutions $u(x, t)$ of the associated parabolic equation, in the limit as $\epsilon \rightarrow 0$, have the property that the associated level sets $\Sigma_{t} \equiv\left\{x \in \mathbf{R}^{n}: u(x, t)=0\right\}$ are evolving by mean curvature flow (cf. $\left[\mathrm{ES}_{*}\right],\left[\mathrm{CGG}_{*}\right],[\mathrm{E}]$ and $[\mathrm{Gi}]$.)

(iv) $f=\operatorname{tr}\{\arctan A\}$. When $X=\mathbf{R}^{n}$, solutions $u(x, t)$ have the property that the graphs of the gradients: $\Gamma_{t} \equiv\{(x, y) \in$ $\left.\mathbf{R}^{n} \times \mathbf{R}^{n}=\mathbf{C}^{n}: y=D_{x} u(x, t)\right\}$ are Lagrangian submanifolds which evolve the initial data by mean curvature flow. (See $[\mathrm{CCH}]$.) 
Techniques discussed above show that:

Comparison holds for the subequation $H_{F}$ on $X \times \mathbf{R}$.

Applying standard viscosity techniques for parabolic equations, one can prove more. Consider a compact subset $K \subset\{t \leq T\} \subset X \times \mathbf{R}$ and let $K_{T} \equiv K \cap\{t=T\}$ denote the terminal time slice of $K$. Let $\partial_{0} K \equiv \partial K-\operatorname{Int} K_{T}$ denote the parabolic boundary of $K$. Here Int $K$ denotes the relative interior in $\{t=T\} \subset X \times \mathbf{R}$. We say that parabolic comparison holds for $H_{F}$ if for all such $K$ (and $T$ )

$$
u+v \leq c \text { on } \partial_{0} K \Rightarrow u+v \leq c \text { on } \operatorname{Int} K
$$

for all $u \in H_{F}(K)$ and $v \in \widetilde{H}_{F}(K)$. Then one has that:

Parabolic comparison holds for the subequation $H_{F}$ on $X \times \mathbf{R}$.

Under further mild assumptions on $f$ which are satisfied in the examples above, one also has existence results. Consider a domain $\Omega \subset X$ whose boundary is strictly $F$ - and $\widetilde{F}$-convex. Set $K=\bar{\Omega} \times[0, T]$. Then

For each $\varphi \in C\left(\partial_{0} K\right)$ there exists a unique function $u \in C(K)$ such that

$$
\left.u\right|_{\operatorname{Int} K} \text { is } H_{F} \text {-harmonic and }\left.u\right|_{\partial_{0} K}=\varphi \text {. }
$$

One then obtains corresponding long-time existence results.

8.6. Obstacle Problems. The methods discussed here lend themselves easily to solving boundary value problems with obstacles. Suppose that $F=\mathbf{R} \times F_{0}$ is a reduced subequation, i.e., independent of the $r$ variable. Given $g \in C(X)$, the associated obstacle subequation is defined to be

$$
H \equiv\left(\mathbf{R}_{-}+g\right) \times F_{0} \quad \text { where } \mathbf{R}_{-} \equiv\{r \leq 0\} \subset \mathbf{R} .
$$

The following facts are easy to prove.

- The $H$-subharmonic functions are the $F$-subharmonic functions $u$ which satisfy $u \leq g$.

- If $F$ has a monotonicity cone $M=\mathbf{R} \times M_{0}$, then $M_{-} \equiv \mathbf{R}_{-} \times M_{0}$ is a monotonicity cone for $H$.

- If $X$ carries a strictly $M$-subharmonic function $\psi$, then on any given compact set, the function $\psi-c$ is strictly $\left(M_{-}\right)$-subharmonic for $c>0$ sufficiently large.

- If $F$ is locally affinely jet-equivalent to a constant coefficient reduced subequation $\mathbf{R} \times \mathbf{F}_{0}$, then $H$ is locally affinely jet-equivalent to the subequation $\mathbf{R}_{-} \times \mathbf{F}_{0}$.

Consequently, under the assumptions in Theorem 8.1.2 on a reduced subequation $F=\mathbf{R} \times F_{0}$ with monotonicity cone $M=\mathbf{R} \times M_{0}$, comparison holds for each associated obstacle subequation $H \equiv\left(\mathbf{R}_{-}+g\right) \times F_{0}$.

However, existence fails for a boundary function $\varphi \in C(\partial \Omega)$ unless $\varphi \leq\left. g\right|_{\partial \Omega}$. Nevertheless, if $\partial \Omega$ is both $F$ and $\widetilde{F}$ strictly convex as in 


\section{Theorem 8.1.2, then existence holds for each boundary function} $\varphi \leq\left. g\right|_{\partial \Omega}$.

To see that this is true, note the following. The Perron family for a boundary function $\varphi \in C(\partial \Omega)$ consists of those $F$-subharmonic functions $u$ on $\Omega$ with $\left.u\right|_{\partial \Omega} \leq \varphi$ (the usual family for $F$ ) subject to the additional constraint $u \leq g$ on $\Omega$. The dual subequation to $H$ is $\widetilde{H}=\left[\left(\mathbf{R}_{-}-g\right) \times\right.$ $\left.J_{\text {red }}^{2}(X)\right] \cup \widetilde{F}$ so that the boundary $\partial \Omega$ is strictly $\widetilde{H}$-convex if it is strictly $\widetilde{F}$-convex. Although $\partial \Omega$ can never be strictly $H$-convex (since $\left(\overrightarrow{F_{\lambda}}\right)_{x}=\emptyset$ for $\lambda>g(x)$ ), the only place that this hypothesis is used in proving Theorem 8.1.2 for $H$ is in the barrier construction which appears in the proof of Proposition $F$ in $\left[\mathrm{HL}_{6}\right]$. However, if $\varphi\left(x_{0}\right) \leq g\left(x_{0}\right)$, then the barrier $\beta(x)$ as defined in (12.1) in $\left[\mathrm{HL}_{6}\right]$ is not only $F$-strict near $x_{0}$ but also automatically $H$-strict since $\beta<g$.

The obstacle problem for the basic subequation $\mathcal{P}$ is related to convex envelopes. This was discovered by Oberman $[\mathrm{O}]$ and developed by ObermanSilvestre [OS].

\section{Restriction Theorems}

Let $F \subset J^{2}(Z)$ be a subequation on a manifold $Z$, and suppose $i: X \subset Z$ is a submanifold. Then there is a natural induced subequation $i^{*} F$ on $X$ given by restriction of 2 -jets. For functions $u \in C^{2}(Z)$ one has directly that

$u$ is $F$-subharmonic on Z $\left.\Rightarrow u\right|_{X}$ is $i^{*} F$-subharmonic on $X$.

Generically this induced subequation $i^{*} F$ is trivial, i.e., all of $J^{2}(X)$. The first problem is to determine the class of submanifolds for which the restriction is interesting. In such cases we then have the following

Question: When does the implication above hold for all $u \in \operatorname{USC}(Z)$ ?

Example. The situation is illustrated by the basic subequation $\mathcal{P}$ in $\mathbf{R}^{n}$ whose subharmonics are the convex functions. The restriction of a smooth convex function $u \in C^{\infty}\left(\mathbf{R}^{n}\right)$ to the unit circle in $\mathbf{R}^{2}$ obeys no proper subequation, while the restriction of $u$ to a minimal submanifold $M \subset \mathbf{R}^{n}$, of any dimension, is subharmonic for the Laplace-Beltrami operator on $M$. This assertion carries over to general convex functions $u$.

9.1. The First General Theorem. The paper [HL $\mathrm{HL}_{9}$ establishes two restriction theorems of a general nature, each of which has interesting applications. The first entails the following technical hypothesis. Fix coordinates $z=(x, y)$ on $Z$ so that locally $X \cong\left\{y=y_{0}\right\}$.

The Restriction Hypothesis: Given $x_{0} \in X$ and $\left(r_{0}, p_{0}, A_{0}\right) \in \mathbf{J}_{n}^{2}$ and given $z_{\epsilon}=\left(x_{\epsilon}, y_{\epsilon}\right)$ and $r_{\epsilon}$ for a sequence of real numbers $\epsilon$ converging to 0 :

$$
\text { If }\left(r_{\epsilon},\left(p_{0}+A_{0}\left(x_{\epsilon}-x_{0}\right), \frac{y_{\epsilon}-y_{0}}{\epsilon}\right),\left(\begin{array}{cc}
A_{0} & 0 \\
0 & \frac{1}{\epsilon} I
\end{array}\right)\right) \in F_{z_{\epsilon}}
$$




$$
\text { and } x_{\epsilon} \rightarrow x_{0}, \quad \frac{\left|y_{\epsilon}-y_{0}\right|^{2}}{\epsilon} \rightarrow 0, \quad r_{\epsilon} \rightarrow r_{0}
$$

then

$$
\left(r_{0}, p_{0}, A_{0}\right) \in\left(i^{*} F\right)_{x_{0}} .
$$

TheOrem 9.1.1. Suppose $u \in \mathrm{USC}(Z)$. Assume the restriction hypothesis and suppose that $\left(i^{*} F\right)$ is closed. Then

$$
\left.u \in F(Z) \quad \Rightarrow \quad u\right|_{X} \in\left(i^{*} F\right)(X) .
$$

If $\left(i^{*} F\right)$ is not closed, the conclusion holds with $\left(i^{*} F\right)$ replaced by $\overline{\left(i^{*} F\right)}$.

9.2. Applications of the First General Theorem. Theorem 9.1.1 applies to several interesting cases. In the following, the term restriction holds refers to the conclusion of Theorem 9.1.1. The reader is referred to $\left[\mathrm{HL}_{9}\right]$ for full statements and proofs.

THEOREM 9.2.1. Let $\mathbf{F}$ be a constant coefficient subequation in $\mathbf{R}^{n}$. Then restriction holds for all affine subspaces $X$ for which $i^{*} \mathbf{F}$ is closed.

More generally, if $u$ is $\mathbf{F}$-subharmonic, then $\left.u\right|_{X}$ is $\overline{i^{*} \mathbf{F}}$-subharmonic.

Consider now a second-order linear operator $\mathbb{L}$ with smooth coefficients on $\mathbf{R}^{n}$. Fix linear coordinates $z=(x, y)$ and suppose $X \cong\left\{y=y_{0}\right\}$ as above. Using the summation convention, write

$\mathbb{L}(u)=A_{i j}(z) u_{x_{i} x_{j}}+a_{i}(z) u_{x_{i}}+\alpha(z) u+B_{k \ell}(z) u_{y_{k} y_{\ell}}+b_{k}(z) u_{y_{k}}+C_{i k}(z) u_{x_{i} y_{k}}$

Suppose the subequation $L$ corresponding to $\mathbb{L} u \geq 0$ satisfies positivity. If any one of the coefficients $B\left(x_{0}, y_{0}\right), b\left(x_{0}, y_{0}\right)$ or $C\left(x_{0}, y_{0}\right)$ is non-zero, restriction is trivial locally since $i^{*} L$ is everything for $x$ near $x_{0}$. Hence, we assume the following

$$
B\left(x, y_{0}\right), b\left(x, y_{0}\right) \text {, and } C\left(x, y_{0}\right) \text { vanish identically on } X
$$

THEOREM 9.2.2. Assuming (9.2.1), restriction holds for the linear operator $L$ to $X$.

This result for linear operators proves to be quite useful.

The next result concerns geometric subequations (see Example 2.1.11) on general riemannian manifolds $Z$.

TheOrem 9.2.3. Let $\mathbb{G} \subset G(p, T Z)$ be a closed subset of the bundle of tangent p-planes on $Z$, which admits a fibre-wise neighborhood retract ( $a$ subbundle for example). Let $F(\mathbb{G})$ be the induced subequation on $Z$, defined as in (2.1.6) using the riemannian hessian. Then restriction holds for all minimal $\mathbb{G}$-submanifolds $X \subset Z$, i.e., minimal submanifolds with $T_{x} X \in \mathbb{G}_{x}$ for all $x \in X$. 
9.3. The Second General Theorem. Let $F$ be a subequation on a manifold $Z$ and fix a submanifold $i: X \subset Z$ as above. In 3.2 .3 we defined the notion of $F$ being locally jet-equivalent to a constant coefficient subequation $\mathbf{F}$. In our current situation there is a notion of $F$ being locally jet-equivalent to $\mathbf{F}$ relative to the submanifold $X$. This entails $i^{*} F$ being locally jet-equivalent to a constant coefficient subequation (assumed closed) on $X$. For details, see $\left[\mathrm{HL}_{9}, \S \S 9\right.$ and 10$]$.

THEOREM 9.3.1. If $F$ is locally jet-equivalent to a constant coefficient subequation relative to $X$, then restriction holds for $F$ to $X$.

9.4. Applications of the Second General Theorem. A nice application of Theorem 9.3.1 is the following.

TheOrem 9.4.1. Let $Z$ be a riemannian manifold of dimension $n$ and $F \subset J^{2}(Z)$ a subequation canonically determined by an $O(n)$-invariant constant coefficient subequation $\mathbf{F} \subset \mathbf{J}^{2}$. Then restriction holds for $F$ to any totally geodesic submanifold $X \subset Z$.

Suppose now that $Z$ is a riemannian manifold with a topological $G$ structure and $F \subset J^{2}(Z)$ is determined by a $G$-invariant constant coefficient subequation as in Section 8.2. The local framings $e_{\alpha}$ appearing in Definition 8.2.1 are called admissible. So also is any framing of the form $e_{\alpha}^{\prime}=g e_{\alpha}$ for a smooth map $g: U_{\alpha} \cap U_{\beta} \rightarrow G$. A submanifold $X \subset Z$ is said to be compatible with the $G$-structure if at every point $z \in X$ there is an admissible framing $e$ on a neighborhood $U$ of $z$ such that on $X \cap U$

$$
\begin{aligned}
& e_{1}, \ldots, e_{n} \text { are tangent to } X \cap U \text { and } \\
& e_{n+1}, \ldots, e_{N} \text { are normal to } X \cap U .
\end{aligned}
$$

For example, if $G=\mathrm{U}(N / 2)$, then any submanifold of constant CR-rank is compatible.

TheOREM 9.4.2. Let $Z$ be a riemannian manifold with topological $G$ structure, and $F \subset J^{2}(Z)$ a subequation canonically determined by a $G$ invariant constant coefficient subequation $\mathbf{F} \subset \mathbf{J}^{2}$. Then restriction holds for $F$ to any totally geodesic submanifold $X \subset Z$ which is compatible with the $G$-structure.

There is a further application of Theorem 9.3.1 to almost complex manifolds, which is discussed in $\S 11$.

\section{Convex Subequations and the Strong Bellman Principle}

An elementary fact, known to all, is that a closed convex set in in a vector space $V$ is the intersection of the closed half-spaces containing it. Put this into a family and you have a fundamental principle, which we call the Bellman Principle, for dealing with nonlinear pde's which are convex. Specifically, suppose $F \subset J^{2}(X)$ is a convex subequation-one with the property that every fibre $F_{x}$ is convex. Then, under mild assumptions, $F$ 
can be written locally as the intersection of a family of linear subequations. These are subequations of the form

$$
L u=\left\langle a, D^{2} u\right\rangle+\langle b, D u\rangle+c u \geq \lambda,
$$

where, from the Conditions $(\mathrm{P})$ and $(\mathrm{N})$ for $F$, one can deduce that the matrix function $a$ and the scalar function $c$ satisfy

$$
a \geq 0 \quad \text { and } \quad c \leq 0 .
$$

The introduction of these local linear equations goes back to Richard Bellman and his work in dynamic programing. These equations can be found in many areas of mathematics. Examples close in spirit to those above appear in work of Bedford-Taylor [BT* ${ }_{*}$ and Krylov $[\mathrm{K}]$.

It is obviously a big improvement if all the linear equations in (10.1) needed to carve out $F$ can be taken to have

$$
a>0,
$$

for then the machinery of uniformly elliptic linear equations can be brought to bear.

More specifically: any $F$-subharmonic function $u$ is locally a viscosity subsolution of $L u \geq \lambda$. From this one sees that $u$ is a classical subsolution (see $\left[\mathrm{HL}_{10}\right.$, Thm. A.5]), and if $a>0$, the results of [HH] apply to prove that $u$ is $L_{\text {loc }}^{1}$. It can then be shown that $u$ is a distributional subsolution to $L u \geq \lambda$, and the full linear elliptic theory ([ $\left.\mathrm{Ho}_{2}\right]$ or $[\mathrm{G}]$ for example) applies.

This naturally raises the question: What assumptions on $F$ will guarantee that it is cut out by linear equations with $a>0$ ?

This question has two parts. The first concerns only the convex geometry of the fibres $F_{x}$ at each point $x$; in other words, the question for a convex constant coefficient subequaton $\mathbf{F} \subset \mathbf{J}^{2}$. The second only involves the mild regularity condition that a containing half-space for $F_{x}$ extends locally to a linear (variable coefficient) subequation containing $F$.

These questions have been discussed in $[\mathrm{K}]$, and an account has also been given in $\left[\mathrm{HL}_{13}\right]$, where the answer to the first question is given as follows. We say that a subset $C \subset \operatorname{Sym}^{2}\left(\mathbf{R}^{n}\right)$ depends on all the variables if there is no proper subspace $W \subset \mathbf{R}^{n}$ and subset $C^{\prime} \subset \operatorname{Sym}^{2}(W)$ such that $\left.A \in C \Longleftrightarrow A\right|_{W} \in C^{\prime}$. Then a (constant coefficient) subequation $\mathbf{F} \subset \mathbf{J}^{2}=\mathbf{R} \times \mathbf{R}^{n} \times \operatorname{Sym}^{2}\left(\mathbf{R}^{n}\right)$ is said to depend weakly on all the second-order variables if for each $(r, p) \in \mathbf{R} \times \mathbf{R}^{n}$, the fibre $\mathbf{F}_{(r, p)}=\{A \in$ $\left.\operatorname{Sym}^{2}\left(\mathbf{R}^{n}\right):(r, p, A) \in \mathbf{F}\right\}$ depends on all the variables.

THEOREM 10.1. If $\mathbf{F}$ depends weakly on all the second-order variables, then $\mathbf{F}$ can be written as the intersection of a family of half-space subequations $\langle a, A\rangle+\langle b, p\rangle+c r \geq \lambda$ with $a>0$.

Note 10.2. For subequations which do not depend on all the second order variables, the conclusions above fail. Consider the (geometrically determined) subequation

$$
\mathbf{F} \cong\left\{u_{x x} \geq 0\right\}
$$


in the $(x, y)$-plane. Any continuous function $u(y)$ is $\mathbf{F}$-subharmonic, in fact, F-harmonic, but not in general $L_{\text {loc }}^{1}$.

See $\left[\mathrm{HL}_{13}\right]$ for a full discussion of these matters.

\section{Applications to Almost Complex Manifolds}

In this section we consider completely general almost complex manifolds $(X, J)$ where $J: T X \rightarrow T X$ is smooth bundle map with $J^{2} \equiv-\mathrm{Id}$. On any such manifold there is an intrinsically defiined subequation

$$
F(J) \subset J^{2}(X),
$$

for which, when the structure is integrable, the $F(J)$-subharmonic functions are exactly the standard plurisubharmonic functions. Hence, the results and techniques discussed in this paper apply to give a full-blown potential theory on almost complex manifolds, which extends the classical theory. The consequences are worked out in detail in $\left[\mathrm{HL}_{10}\right]$. Here are a few highlights.

11.1. J-Holomorphic Curves. A submanifold $Y \subset X$ is an almost complex submanifold if $J\left(T_{y} Y\right)=T_{y} Y$ for all $y \in Y$. In general dimensions such submanifolds exist only rarely. However, when the real dimension of $Y$ is two, $Y$ is called a $J$-holomorphic curve, and we have the following important classical result.

THEOREM 11.1.1. (Nijenhuis and Woolf $[N W]$ ) For each point $x \in X$ and each complex tangent line $\ell \subset T_{x} X$, there exists a J-holomorphic curve passing through $x$ with tangent direction $\ell$.

The restriction result 9.3.1 applies in this case to prove the following. For historical compatibility we replace the term " $F(J)$-subharmonic" with " $F(J)$-plurisubharmonic".

THEOREM 11.1.2. Let $\left(Y, J_{Y}\right)$ be an almost complex submanifold of $\left(X, J_{X}\right)$. Then the restriction of any $F\left(J_{X}\right)$-plurisubharmonic function to $Y$ is $F\left(J_{Y}\right)$-plurisubharmonic.

This leads to the following result equating two natural definitions of plurisubharmonicity. We recall that an almost complex structure $J$ on a 2-dimensional manifold $S$ is always integrable, and all notions of (usc) subharmonic functions on $(S, J)$ coincide.

THEOREM 11.1.3. A function $u \in \mathrm{USC}(X)$ is $F(J)$-plurisubharmonic if and only if its restriction to every J-holomorphic curve is subharmonic.

11.2. Completion of the Pali Conjecture. There is a third definition of $J$-plurisubharmonic functions on an almost complex manifold $(X, J)$, which makes sense for any distribution $u \in \mathcal{D}^{\prime}(X)$. Any such distribution $u$ is known to be $L_{\text {loc }}^{1}$. By work of Nefton Pali $[\mathrm{P}]$ we know that any $\in \operatorname{USC}(X)$ which is $J$-plurisubharmonic in the sense of Section 11.1, is $L_{\text {loc }}^{1}$ on $X$ and $J$-plurisubharmonic as a distribution. In the converse direction he showed 
that if a $J$-plurisubharmonic distribution $u$ has a continuous representative (as a $[-\infty, \infty)$-valued function), then it is $J$-plurisubharmonic as above. He further conjectured that the converse should hold in general. This was proved in $\left[\mathrm{HL}_{10}\right]$.

The proof used the Strong Bellman Principle and involved showing that the upper semi-continuous representative of the $L_{\text {loc }}^{1}$-class obtained for each of the associated linear equations, is independent of the linear equation. It is, in fact, given by the essential upper-semi-continuous regularization

$$
u_{\mathrm{ess}}^{*}(x) \equiv \lim _{r \searrow 0}\left\{\operatorname{ess} \sup _{B_{x}(r)} u\right\}
$$

which depends only on the $L_{\text {loc }}^{1}$-class of $u$.

11.3. The Dirichlet Problem for Maximal Functions. Theorem 8.12 applies in this case to prove existence and uniqueness for the Dirichlet problem for $J$-maximal functions. One can show that the more classical notion of a function $u$ being $J$-maximal (going back to $[\mathrm{B}],[\mathrm{W}]$ ), is the same as $u$ being $F(J)$-harmonic, i.e., $u$ is $F(J)$-(pluri)subharmonic and $-u$ is $\widetilde{F}(J)$-subharmonic. A domain $\Omega \subset \subset X$ with smooth boundary is strictly $J$-convex if it has a strictly $F(J)$-plurisubharmonic defining function.

TheOREm 11.3.1. Let $\Omega \subset \subset X$ be a strictly $J$-convex domain in an almost complex manifold $(X, J)$. Then the Dirichlet problem for $J$-maximal functions in uniquely solvable on $\Omega$ for all continuous boundary values $\varphi \in C(\partial \Omega)$.

Note 11.3.2. Recently Szymon Pliś has also studied the Dirichlet problem on almost complex manifolds $[\mathrm{Pl}]$. His result is the almost-complex analogue of a main result in [CKNS]. It treats the inhomogeneous MongeAmpère equation with positive right hand side. All data are assumed to be smooth, and complete regularity is established for the solution.

\section{Appendix A. A Pocket Dictionary}

The conventions adopted in this paper (and related ones) are not common in the literature, but they have several advantages, particularly for applications to calibrated geometry and to branches of polynomial operators. In the case of comparison the advantage is discussed in Comment 3 below.

For readers hard-wired to standard notation (as in, say, [CIL]), we give here a concise translation of concepts to serve as a guide.

Classically, a fully nonlinear partial differential equation for a smooth function $u(x)$ on an open set $X \subset \mathbf{R}^{n}$ is written in the form

$$
f\left(x, u, D u, D^{2} u\right)=0
$$

for a given contiinuous function $f: X \times \mathbf{R} \times \mathbf{R}^{n} \times \operatorname{Sym}^{2}\left(\mathbf{R}^{n}\right) \longrightarrow \mathbf{R}$.

Here the function $f$ is typically replaced by the closed set

$$
F \equiv\{(x, r, p, A): f(x, r, p, A) \geq 0\} .
$$


For $C^{2}$-functions $u(x)$ we have the following translations. Set $J_{x}^{2} u \equiv$ $\left(x, u, D u, D^{2} u\right)$.

$$
\begin{aligned}
& u \text { is a subsolution } \quad \longrightarrow \quad u \text { is } F \text { subharmonic, i.e., } \\
& f\left(x, u, D u, D^{2} u\right) \geq 0 \quad \longrightarrow \quad J_{x}^{2} u \in F \quad \forall x \in X . \\
& u \text { is a supersolution } \quad \longrightarrow \quad-u \text { is } \widetilde{F} \text { subharmonic, i.e., } \\
& f\left(x, u, D u, D^{2} u\right) \leq 0 \quad \longrightarrow \quad-J_{x}^{2} u \in \widetilde{F} \quad \forall x \in X . \\
& u \text { is a solution } \quad \longrightarrow \quad u \text { is } F \text { harmonic, i.e., } \\
& f\left(x, u, D u, D^{2} u\right)=0 \quad \longrightarrow \quad J_{x}^{2} u \in \partial F \quad \forall x \in X \\
& \longleftrightarrow \quad u \text { is } F \text { subharmonic and } \\
& -u \text { is } \widetilde{F} \text { subharmonic }
\end{aligned}
$$

These same translations apply to any upper semi-continuous function $u$ by applying them to test functions at each point $x$.

We also have the following translations between some of the standard structural conditions placed on the function $f$ and conditions on the set $F$. Let $\mathcal{P} \equiv\{(0,0, A): A \geq 0\}$ and $\mathcal{N} \equiv\{(r, 0,0): r \leq 0\}$.

$$
\begin{aligned}
f \text { is degenerate elliptic } & \longrightarrow F \text { satisfies positivity, i.e., } \\
f(x, r, p, A+P) \geq f(x, r, p, A) \forall P \geq 0 & \longrightarrow F+\mathcal{P} \subset F . \\
f \text { is monotone in the dependent variable } & \longrightarrow \\
F \text { satisfies negativity, i.e., } & \longrightarrow \\
f(x, r-s, p, A) \geq f(x, r, p, A) \forall s \geq 0 & \longrightarrow F+\mathcal{N} \subset F .
\end{aligned}
$$

$f$ is proper if both conditions hold $\longrightarrow F+\mathcal{P} \subset F$ and $F+\mathcal{N} \subset F$

$f$ is uniformly elliptic $\longleftrightarrow\left\{\begin{array}{c}F+\mathbf{P}_{\lambda, \Lambda} \subset F \text { for some } 0<\lambda<\Lambda, \\ \text { or equivalently, } \\ F+\mathbf{P}(\delta) \subset F \text { for some } \delta>0 .\end{array}\right.$

Here $\mathbf{P}_{\lambda, \Lambda}$ is the Pucci cone discussed in $\S 4.5$, and $\mathcal{P}(\delta)$ is the cone defined in Example 4.3.3.

It is important to realize that these translations are not precise equivalences (although there is an implication). In passing from the function $f$ to the set $F \equiv\{f \geq 0\}$, the behavior of $f$ away from its zero-set is lost. Matters become simpler, and this can be an advantage (See Comment 3). There are also natural examples where the set $\{f \geq 0\}$ is not really what one wants to take for the set $F$, and the topological condition required in the "set" point of view easily corrects matters (see Comment 2 below). 
Comment 1. As noted above, these translations are not equivalences in general. For example, the positivity condition $F+\mathcal{P} \subset F$ is equivalent to the assumption that

$$
f(x, r, p, A) \geq 0 \quad \Rightarrow \quad f(x, r, p, A+P) \geq 0 \quad \forall P \geq 0 .
$$

which is weaker than the inequality on $f$ required for degenerate ellipticity. The negativity condition $F+\mathcal{N} \subset F$ is equivalent to the assumption that

$$
f(x, r, p, A) \geq 0 \quad \Rightarrow \quad f(x, r-s, p, A) \geq 0 \quad \forall s \geq 0 .
$$

which is weaker than the properness condition placed on $f$ above.

Comment 2. The Topological Condition (T) that $F=\overline{\operatorname{Int} F}$, holds for most classical equations of interest. However, there are cases where it fails, such as the infinite Laplaican $f(p, A)=\langle A p, p\rangle$ or the $k$-Laplacian $|p|^{2}+(k-2)\langle A p, p\rangle,(1 \leq k \neq 2)$. When it fails, it is condition (T) that selects the "correct" subequation $F$.

Comment 3 (Supersolutions versus $\widetilde{F}$-subharmonicity). There is an important difference between $u$ being a supersolution and $-u$ being $\widetilde{F}$ subharmonic, which arises when $\operatorname{Int} F \neq\{f>0\}$. However, since we have $\{f>0\} \subset \operatorname{Int} F$ (equivalently $\sim \operatorname{Int} F \subset\{f \leq 0\}$ ) we deduce

$$
-v \text { is } \widetilde{F} \text { subharmonic } \quad \Rightarrow \quad v \text { is an } f \text { supersolution. }
$$

The fact that the converse is not true is important. For a constant coefficient, pure second-order subequation $F \subset \operatorname{Sym}^{2}\left(\mathbf{R}^{n}\right)$, the more restrictive condition on $v$ in (A.1) ensures that comparison holds. That is, with $u F$ subharmonic and $-v \widetilde{F}$-subharmonic,

$$
u \leq v \text { on } \partial K \Rightarrow u \leq v \text { on } K
$$

(See $\left[\mathrm{HL}_{4}\right]$ for a proof.) One can show that (A.1) is an equivalence if and only if whenever $F(A)=0$, the function $F(A+\epsilon I)$ has an isolated zero at $\epsilon=0$.

\section{Appendix B. Examples of Basic Monotonicity Cones}

The following is a list of constant-coefficient convex cone subequations $\mathbf{M}$ such that the Zero Maximum Principle (see §4.1) holds for $\widetilde{\mathbf{M}}$-subharmonic functions. In cases (1), (5) and (6) the full maximum principle holds, since these equations are independent of the $r$-variable.

(1) $\mathbf{M}=\mathbf{R} \times \mathbf{R}^{n} \times \mathcal{P}$. Here the $\widetilde{\mathbf{M}}$-subharmonic functions are the subaffine functions (see Proposition 2.1.7). This is a monotonicity subequation for any pure second-order subequation $\mathbf{F}=\mathbf{R} \times \mathbf{R}^{n} \times \mathbf{F}_{0}$.

(2) $\mathbf{M}=\mathbf{R}_{-} \times \mathbf{R}^{n} \times \mathcal{P}$. Here one can characterize the $\widetilde{\mathbf{M}}$-subharmonics as being "sub" the functions of the form $\max \{0, a(x)\}$ with $a(x)$ affine (the affine-plus functions). This is a monotonicity subequation for any gradientindependent subequation. 
(3) $\mathbf{M}=\mathbf{R}_{-} \times \mathcal{D} \times \mathcal{P}$ with $\mathcal{D} \subset \mathbf{R}^{n}$ a "directional" convex cone with vertex at the origin and non-empty interior.

(4) $\mathbf{M}=\left\{(r, p, A) \in \mathbf{J}^{2}: r \leq-\gamma|p|, \quad p \in \mathcal{D}\right.$ and $\left.A \geq 0\right\}$ with $\gamma>0$ and $\mathcal{D} \subset \mathbf{R}^{n}$ as above.

(5) $\mathbf{M}=\mathbf{R} \times \mathbf{M}_{0}$ with $(p, A) \in \mathbf{M}_{0} \Longleftrightarrow\langle A e, e\rangle-\lambda|\langle p, e\rangle| \geq 0 \forall|e|=1$

For the next example the Maximum Principle only holds for compact sets $K \subset \mathbf{R}^{n}$ which are contained in a ball of radius $R$.

(6) $\quad \mathbf{M}=\mathbf{R} \times \mathbf{M}_{0} \quad$ with $(p, A) \in \mathbf{M}_{0} \Longleftrightarrow A-\frac{|p|}{R} \mathrm{Id} \geq 0$

The proofs depend on the following elementary result.

TheOrem B.2. Suppose $\mathbf{M}$ is a constant coefficient convex subequation and $K \subset \mathbf{R}^{n}$ is compact. If $K$ admits a smooth function $\psi$ which is strictly M-subharmonic on Int $K$, then the Zero Maximum Principle holds for the dual subequation $\widetilde{\mathbf{M}}$ on $K$.

Proof. Suppose that the (ZMP) fails for $u \in \operatorname{USC}(K)$. We will show that there exists a point $\bar{x} \in \operatorname{Int} K$ and $\epsilon>0$ such that $\varphi \equiv-\epsilon \psi$ is a test function for $u$ at $\bar{x}$. This proves that $u$ is not $\widetilde{\mathbf{M}}$-subharmonic near $\bar{x}$ because $J_{\bar{x}}^{2} \psi \in$ IntM implies that $J_{\bar{x}}^{2} \varphi=-\epsilon J_{\bar{x}}^{2} \psi \notin \widetilde{\mathbf{M}}$.

By assumption, $u \leq 0$ on $\partial K$ but $\sup _{K} u>0$. The negativity condition (N) for $\widetilde{\mathbf{M}}$ allows us to subtract a small number from $u$ and assume that $u<0$ on $\partial K$ with $\sup _{K} u>0$. Set $v \equiv u+\epsilon \psi$. Then with $\epsilon>0$ sufficiently small, $v<0$ on $\partial K$ but $\sup _{K} v>0$. Now let $\bar{x}$ denote a maximum point for $v$ on $K$. Since $\bar{x} \in \operatorname{Int} K$, this proves that $\varphi \equiv-\epsilon \psi$ is a test function for $u$ at $\bar{x}$ as desired.

Proof of (1)-(4). Since the $\mathbf{M}$ in (4) is contained in the other three M's, it suffices to find a strictly $\mathbf{M}$-subharmonic function for $\mathbf{M}$ defined as in (4). Choose $\psi(x) \equiv \frac{1}{2} \delta\left|x-x_{0}\right|^{2}-c$ with $\delta, c>0$. Denote the jet coordinates of $\psi$ at $x \in K$ by $r=\psi(x), p=\delta\left(x-x_{0}\right)$ and $A=\delta I$. Choose $x_{0} \in \mathbf{R}^{n}$ so that $K \subset x_{0}+\operatorname{Int} \mathcal{D}$. Then $A \in \operatorname{Int} \mathcal{P}, p \in \operatorname{Int} \mathcal{D}$ and $r+\gamma|p|=\frac{1}{2} \epsilon\left|x-x_{0}\right|^{2}-c+\gamma \delta\left|x-x_{0}\right|<0$ if $c$ is large.

Proof of (5). Consider $\psi(x) \equiv \frac{1}{N+1}|x|^{N+1}$. Then one computes that

$$
p=D \psi=|x|^{N} \frac{x}{|x|} \quad \text { and } \quad A=D^{2} \psi=|x|^{N-1}\left(I+(N-1) P_{[x]}\right)
$$

where $P_{[x]}$ is orthogonal projection onto the $x$-line. Then with $|e|=1$ we have

$$
\frac{1}{|x|^{N-1}}(\langle A e, e\rangle-\lambda|\langle p, e\rangle|)=1-\lambda|x| t+(N-1) t^{2} \equiv g(t) .
$$

with $t \equiv\left|\left\langle\frac{x}{|x|}, e\right\rangle\right|$. We can assume that $0 \notin K$ and $x \in K$ implies $|x| \leq R$. The quadratic $g(t)$ has a minimum at $t_{0}=\frac{\lambda|x|}{2(N-1)}$ with the minimum value 
$g\left(t_{0}\right)=1-\frac{\lambda^{2}|x|^{2}}{4(N-1)} \geq 1-\frac{\lambda^{2} R^{2}}{4(N-1)}$. Choose $N$ large enough so that this is $>0$.

Proof of (6). This is similar to the proof of (5). It reduces to showing that $g(t)=1-\frac{|x|}{R}+(N-1) t^{2}>0$. Now the minimum value (at $\left.t=0\right)$ is $1-\frac{|x|}{R}$. For the counterexample, consider

$$
u(x) \equiv\left\{\begin{array}{cc}
-(R-|x|)^{3} & |x| \leq R \\
0 & |x| \geq R
\end{array}\right.
$$

\section{References}

$\left[\mathrm{A}_{1}\right] \quad$ S. Alesker, Non-commutative linear algebra and plurisubharmonic functions of quaternionic variables, Bull. Sci. Math., 127 (2003), 1-35. also ArXiv:math.CV/0104209.

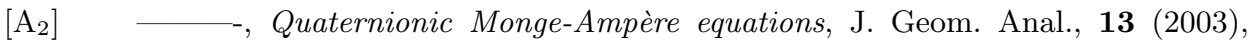
205-238. ArXiv:math.CV/0208805.

[AV] S. Alesker and M. Verbitsky, Plurisubharmonic functions on hypercomplex manifolds and HKT-geometry, arXiv: math.CV/0510140 Oct.2005

[Al] A. D. Alexandrov, The Dirichlet problem for the equation Det $\left\|z_{i, j}\right\|=$ $\psi\left(z_{1}, \ldots, z_{n}, x_{1}, \ldots, x_{n}\right)$, I. Vestnik, Leningrad Univ. 13 No. 1, (1958), 5-24.

[AGV] M. E. Amendola, G. Galise and A. Vitolo, Riesz capacity, maximum principle and removable sets of fully nonlinear second-order elliptic operators, Preprint, University of Salerno.

[AFS] D. Azagra, J. Ferrera and B. Sanz, Viscosity solutions to second order partial differential equations on riemannian manifolds, ArXiv:math.AP/0612742v2, Feb. 2007.

$\left[\mathrm{BT}_{1}\right] \quad$ E. Bedford and B. A. Taylor, The Dirichlet problem for a complex MongeAmpère equation, Inventiones Math.37 (1976), no.1, 1-44.

$\left[\mathrm{BT}_{2}\right] \longrightarrow$, Variational properties of the complex Monge-Ampère equation, I. Dirichlet principle, Duke Math. J. 45 (1978), no. 2, 375-403.

$\left[\mathrm{BT}_{3}\right] \quad-$, A new capacity for plurisubharmonic functions, Acta Math.149 (1982), no.1-2, 1-40.

[B] H. J. Bremermann, On a generalized Dirichlet problem for plurisubharmonic functions and pseudo-convex domains, Trans. A. M. S. 91 (1959), 246-276.

[BH] R. Bryant and F. R. Harvey, Submanifolds in hyper-Kähler geometry, J. Amer. Math. Soc. 1 (1989), 1-31.

[CKNS] L. Caffarelli, J. J. Kohn, L. Nirenberg, and J. Spruck, The Dirichlet problem for non-linear second order elliptic equations II: Complex Monge-Ampère and uniformly elliptic equations, Comm. on Pure and Applied Math. 38 (1985), 209252.

[CLN] L. Caffarelli, Y.Y. Li and L. Nirenberg Some remarks on singular solutions of nonlinear elliptic equations, III: viscosity solutions, including parabolic operators. ArXiv:1101.2833.

$\left[\mathrm{CNS}_{1}\right]$ L. Caffarelli, L. Nirenberg and J. Spruck, The Dirichlet problem for nonlinear second order elliptic equations. I: Monge-Ampère equation, Comm. Pure Appl. Math. 37 (1984), 369-402.

$\left[\mathrm{CNS}_{2}\right] \longrightarrow$ - The Dirichlet problem for nonlinear second order elliptic equations, III: Functions of the eigenvalues of the Hessian, Acta Math. 155 (1985), 261-301.

[CNS 3$]_{-}^{-}$, The Dirichlet problem for the degenerate Monge-Ampère equation, Rev. Mat. Iberoamericana 2 (1986), 19-27. 
$\left[\mathrm{CNS}_{4}\right] \longrightarrow-$ Correction to: "The Dirichlet problem for nonlinear second order elliptic equations. I: Monge-Ampère equation", Comm. Pure Appl. Math. 40 (1987), 659-662.

[CCH] A. Chau, J. Chen and W. He, Lagrangian mean curvature flow for entire Lipschitz graphs, ArXiv:0902.3300 Feb, 2009.

$\left[\mathrm{CGG}_{1}\right]$ Y.-G. Chen, Y. Giga and S. Goto, Uniqueness and existence of viscosity solutions of generalized mean curvature flow equations, Proc. Japan Acad. Ser. A. Math. Sci 65 (1989), 207-210.

$\left[\mathrm{CGG}_{2}\right] \longrightarrow$ - Uniqueness and existence of viscosity solutions of generalized mean curvature flow equations, J. Diff. Geom. 33 (1991), 749-789.

$\left[\mathrm{CY}_{1}\right] \quad$ S.-Y. Cheng and S.-T. Yau, On the regularity of the Monge-Ampère equation $\operatorname{det}\left(\partial^{2} u / \partial x_{i} \partial x_{j}\right)=F(x, u)$, Comm. Pure Appl. Math. 30 (1977), no. 1, 41-68.

$\left[\mathrm{CY}_{2}\right] \quad-$, The real Monge-Ampère equation and affine flat structures, Proceedings of the 1980 Beijing Symposium on Differential Geometry and Differential Equations, Vol. 1, 2, 3 (Beijing, 1980), 339-370, Science Press, Beijing, 1982.

[C] M. G. Crandall, Viscosity solutions: a primer, pp. 1-43 in "Viscosity Solutions and Applications" Ed.'s Dolcetta and Lions, SLNM 1660, Springer Press, New York, 1997.

[CIL] M. G. Crandall, H. Ishii and P. L. Lions User's guide to viscosity solutions of second order partial differential equations, Bull. Amer. Math. Soc. (N. S.) 27 (1992), 1-67.

[EGZ] P. Eyssidieux, V. Guedj, and A. Zeriahi, Viscosity solutions to degenerate MongeAmpère equations, ArXiv:1007.0076.

[E] L. C. Evans, Regularity for fully nonlinear elliptic equations and motion by mean curvature, pp. 98-133 in "Viscosity Solutions and Applications" Ed.'s Dolcetta and Lions, SLNM 1660, Springer Press, New York, 1997.

[ESm] L. C. Evans and C. K. Smart, Everywhere differentiability of infinite harmonic functions, Berkeley preprint, 2012

$\left[\mathrm{ES}_{1}\right] \quad$ L. C. Evans and J. Spruck, Motion of level sets by mean curvature, I, J. Diff. Geom. 33 (1991), 635-681.

[ES $\mathrm{ES}_{2} \quad-$, Motion of level sets by mean curvature, II, Trans. A. M. S. 330 (1992), $321-332$.

[ES $\mathrm{ES}_{3} \quad-$, Motion of level sets by mean curvature, III, J. Geom. Anal. 2 (1992), $121-150$.

$\left[\mathrm{ES}_{4}\right] \quad-$, Motion of level sets by mean curvature, IV, J. Geom. Anal. 5 (1995), 77-114.

[G] P. Garabedian, Partial Differential Equations, J. Wiley and Sons, New York, 1964.

[G] L. Gårding, An inequality for hyperbolic polynomials, J. Math. Mech. 8 no. 2 (1959), 957-965.

[Gi] Y. Giga, Surface Evolution Equations-A level set approach, Birkhäuser, 2006.

[Giu] E. Giusti, Minimal surfaces and functions of bounded variation, Monographs in Mathematics, 80. Birkhäuser Verlag, Basel, 1984.

[Gr] M. Gromov, Pseudoholomorphic curves in symplectic manifolds, Invent. Math. 82 (1985), no. 2, 307-347.

$[\mathrm{H}] \quad$ F. R. Harvey, Removable singularities and structure theorems for positive currents. Partial differential equations (Proc. Sympos. Pure Math., Vol. XXIII, Univ. California, Berkeley, Calif., 1971), pp. 129-133. Amer. Math. Soc., Providence, R.I., 1973.

$\left[\mathrm{HL}_{1}\right] \quad$ F. R. Harvey and H. B. Lawson, Jr, Calibrated geometries, Acta Mathematica 148 (1982), 47-157.

$\left[\mathrm{HL}_{2}\right] \quad-$, An introduction to potential theory in calibrated geometry, Amer. J. Math. 131 no. 4 (2009), 893-944. ArXiv:math.0710.3920. 
$\left[\mathrm{HL}_{3}\right] \longrightarrow$ Duality of positive currents and plurisubharmonic functions in calibrated geometry, Amer. J. Math. 131 no. 5 (2009), 1211-1240. ArXiv:math.0710.3921.

$\left[\mathrm{HL}_{4}\right] \quad$ - Dirichlet duality and the non-linear Dirichlet problem, Comm. on Pure and Applied Math. 62 (2009), 396-443. ArXiv:math.0710.3991

[HL $\mathrm{HL}_{5} \longrightarrow$, Plurisubharmonicity in a general geometric context, Geometry and Analysis 1 (2010), 363-401. ArXiv:0804.1316.

$\left[\mathrm{HL}_{6}\right] \longrightarrow$, Dirichlet duality and the nonlinear Dirichlet problem on Riemannian manifolds, J. Diff. Geom. 88 (2011), 395-482. ArXiv:0912.5220.

$\left[\mathrm{HL}_{7}\right] \longrightarrow$, Hyperbolic polynomials and the Dirichlet problem, ArXiv:0912.5220.

[HL $\mathrm{HL}_{8} \quad$, Gårding's theory of hyperbolic polynomials, to appear in Communications in Pure and Applied Mathematics.

[[HL9] - The restriction theorem for fully nonlinear subequations, Ann. Inst. Fourier (to appear). ArXiv:1101.4850.

$\left[\mathrm{HL}_{10}\right] \longrightarrow$, Potential Theory on almost complex manifolds, Ann. Inst. Fourier (to appear). ArXiv:1107.2584.

$\left[\mathrm{HL}_{11}\right] \longrightarrow$, Foundations of p-convexity and p-plurisubharmonicity in riemannian geometry, ArXiv: 1111.3895.

[HL $\left.\mathrm{HL}_{12}\right]-$, Removable singularities for nonlinear subequations. (Stony Brook Preprint).

$\left[\mathrm{HL}_{13}\right] \longrightarrow$, The equivalence of viscosity and distributional subsolutions for convex subequations - the strong Bellman principle, Bol. Soc. Bras. de Mat. (to appear). ArXiv:1301.4914.

[HL $\left.\mathrm{HL}_{14}\right]$ - Lagrangian plurisubharmonicity and convexity, Stony Brook Preprint.

$\left[\mathrm{HL}_{15}\right] \longrightarrow$, Radial subequations, isolated singularities and tangent functions, Stony Brook Preprint.

[HP 1$] \quad$ F. R. Harvey and J. Polking, Removable singularities of solutions of linear partial differential equations, Acta Math. 125 (1970), 39-56.

$\left[\mathrm{HP}_{2}\right] \quad-$ Extending analytic objects, Comm. Pure Appl. Math. 28 (1975), 701727.

[HH] M. Hervé and R.M. Hervé., Les fonctions surharmoniques dans l'axiomatique de M. Brelot associées à un opérateur elliptique dégénéré, Annals de l'institut Fourier, 22, no. 2 (1972), 131-145.

$\left[\mathrm{Ho}_{1}\right]$ L. Hörmander, An introduction to complex analysis in several variables, Third edition. North-Holland Mathematical Library, 7. North-Holland Publishing Co., Amsterdam, 1990.

$\left[\mathrm{Ho}_{2}\right] \longrightarrow$, The analysis of linear partial differential operators. III. Pseudodifferential operators. Grundlehren der Mathematischen Wissenschaften [Fundamental Principles of Mathematical Sciences], 274. Springer-Verlag, Berlin, 1985.

[HM] L. R. Hunt and J. J. Murray, q-plurisubharmonic functions and a generalized Dirichlet problem, Michigan Math. J., 25 (1978), 299-316.

[I] H. Ishii, On uniqueness and existence of viscosity solutions of fully nonlinear second-order elliptic pde's, Comm. Pure and App. Math. 42 (1989), 14-45.

[IL] H. Ishii and P. L. Lions, Viscosity solutions of fully nonlinear second-order elliptic partial differential equations, J. Diff. Eq. 83 (1990), 26-78.

$\left[\mathrm{J}_{1}\right] \quad \mathrm{R}$. Jensen, Uniqueness criteria for viscosity solutions of fully nonlinear elliptic partial differential equations, Indiana Univ. Math. J. 38 (1989), 629-667.

$\left[\mathrm{J}_{2}\right] \quad-$, Uniqueness of Lipschitz extensions: minimizing the sup norm of the gradient, Arch. Rational Mech. Analysis 123 (1993), 51-74.

[JLM] P. Juutinen, P. Lindqvist, and J. Manfredi, On the equivalence of viscosity solutions and weak solutions for a quasi-linear equation, SIAM J. Math. Anal. 33 (2001), no. 3, 699-717.

[K] N. V. Krylov, On the general notion of fully nonlinear second-order elliptic equations, Trans. Amer. Math. Soc. (3) 347 (1979), 30-34. 
[La $]$ D.Labutin, Isolated singularities for fully nonlinear elliptic equations, J. Differential Equations 177 (2001), No. 1, 49-76.

[ $\left.\mathrm{La}_{2}\right]$ D.Labutin, Singularities of viscosity solutions of fully nonlinear elliptic equations, Viscosity Solutions of Differential Equations and Related Topics, Ishii ed., RIMS Kôkyûroku No. 1287, Kyoto University, Kyoto (2002), 45-57

[La3] - Potential estimates for a class of fully nonlinear elliptic equations, Duke Math. J. 111 No. 1 (2002), 1-49.

[L] N. S. Landkof, Foundations of Modern Potential Theory, Springer-Verlag, New York, 1972.

[Le] P. Lelong, Fonctions plurisousharmoniques et formes différentielles positives, Gordon and Breach, Paris-London-New York (Distributed by Dunod Éditeur, Paris) 1968.

[LE] Y. Luo and A. Eberhard, An application of $C^{1,1}$ approximation to comparison principles for viscosity solutions of curvatures equations, Nonlinear Analysis 64 (2006), 1236-1254.

[NTV] N. Nadirashvili, V. Tkachev and S. Vlùadutü Non-classical Solution to Hessian Equation from Cartan Isoparametric Cubic, ArXiv:1111. 0329.

[NW] A. Nijenhuis and W. Woolf, Some integration problems in almost-complex and complex manifolds, Ann. of Math., 77 (1963), 424-489.

[O] A. Oberman, The convex envelope is the solution of a nonlinear obstacle problem, Proc. A.M.S. 135 (2007), no. 6, 1689-1694.

[OS] A. Oberman and L. Silvestre, The Dirichlet problem for the convex envelope, Trans. A.M.S. 363 (2011), no. 11, 5871-5886.

[P] N. Pali, Fonctions plurisousharmoniques et courants positifs de type $(1,1)$ sur une variété presque complexe, Manuscripta Math. 118 (2005), no. 3, 311-337.

[PZ] S. Peng and D. Zhou, Maximum principle for viscosity solutions on riemannian manifolds, ArXiv:0806.4768, June 2008.

[Pl] S. Pliś, The Monge-Ampère equation on almost complex manifolds, ArXiv:1106.3356, June, 2011.

$\left[\mathrm{Po}_{1}\right] \quad \mathrm{A} . \mathrm{V}$. Pogorelov, On the regularity of generalized solutions of the equation det $\left(\partial^{2} u / \partial x_{i} \partial x_{j}\right)=\phi\left(x_{1}, \ldots, x_{n}\right)>0$, Dokl. Akad. Nauk SSSR 200, 1971, pp. 534537.

$\left[\mathrm{PO}_{2}\right] \quad-$, The Dirichlet problem for the $n$-dimensional analogue of the MongeAmpère equation, Dokl. Akad. Nauk SSSR 201, 1971, pp. 790-793.

[RT] J. B. Rauch and B. A. Taylor, The Dirichlet problem for the multidimensional Monge-Ampère equation, Rocky Mountain J. Math 7 (1977), 345-364.

[Sh ${ }_{1} \quad$ J.-P. Sha, p-convex riemannian manifolds, Invent. Math. 83 (1986), 437-447.

$\left[\mathrm{Sh}_{2}\right] \quad-$, Handlebodies and p-convexity, J. Diff. Geom. 25 (1987), 353-361.

[Shi] B. Shiffman, Extension of positive line bundles and meromorphic maps., Invent. Math. 15 (1972), no. 4, 332-347.

$\left[\mathrm{S}_{1}\right] \quad$ Z. Slodkowski, The Bremermann-Dirichlet problem for q-plurisubharmonic functions, Ann. Scuola Norm. Sup. Pisa Cl. Sci. (4) 11 (1984), 303-326.

$\left[\mathrm{S}_{2}\right] \quad-$ Pseudoconvex classes of functions. I. Pseudoconcave and pseudoconvex sets, Pacific J. of Math. 134 no. 2 (1988), 343-376.

[S $\left.\mathrm{S}_{3}\right] \quad-$ Pseudoconvex classes of functions. II. Affine pseudoconvex classes on $\mathbf{R}^{N}$, Pacific J. of Math. 141 no. 1 (1990), 125-163.

$\left[\mathrm{S}_{4}\right] \quad-$ Pseudoconvex classes of functions. III. Characterization of dual pseudoconvex classes on complex homogeneous spaces, Trans. A. M. S. 309 no.1 (1988), 165-189.

[S5] - Complex interpolation of normed and quasinormed spaces in several dimensions, I. Trans. Amer. Math. Soc. 308 (1988), no. 2, 685-711.

$\left[\mathrm{SYZ}_{1}\right]$ A. Strominger, S.-T. Yau and E. Zaslow, Mirror symmetry is T-duality, Winter School on Mirror Symmetry, Vector Bundles and Lagrangian Submanifolds 
(Cambridge, MA, 1999), 333-347, AMS/IP Stud. Adv. Math., 23, Amer. Math. Soc., Providence, RI, 2001.

[SYZ $\left.{ }_{2}\right]-$, Mirror symmetry is T-duality, Nuclear Phys. B 479 (1996), no. 1-2, 243-259.

[So] P. Soravia, On nonlinear convolution and uniqueness of viscosity solutions, Analysis 20 (2000), 373-386.

[TU] N. S. Trudinger and J.n I. E. Urbas, Second derivative estimates for equations of Monge-Ampère type, Bull. Austral. Math. Soc. 30 (1984), 321-334.

[W] J. B. Walsh, Continuity of envelopes of plurisubharmonic functions, J. Math. Mech. 18 (1968-69), 143-148.

[WY] D. Wang and Y. Yuan, Hessian estimates for special Lagrangian equation with critical and supercritical phases in general dimensions, ArXiv:1110.1417.

[Wu] H. Wu, Manifolds of partially positive curvature, Indiana Univ. Math. J. 36 No. 3 (1987), 525-548.

[Yau] S.-T. Yau, On the Ricci curvature of a compact Köhler manifold and the complex Monge-Ampère equation. I, Comm. Pure Appl. Math. 31 (1978), no. 3, 339-411.

[Y] Yu Yuan, A priori estimates for solutions of fully nonlinear special lagrangian equations, Ann Inst. Henri Poincaré 18 (2001), 261-270.

Mathematics Department, Rice University, Houston, TX 77005-1982, USA

E-mail address: harvey@rice.edu

Mathematics Department, Stony Brook University, Stony Brook, Ny 11790-3651, USA

E-mail address: blaine@math.sunysb.edu 